\title{
Nonlinear fluctuations in relativistic causal fluids
}

\author{
Nahuel Miron-Granese, ${ }^{a}$ Alejandra Kandus ${ }^{b}$ and Esteban Calzetta ${ }^{c, d}$ \\ ${ }^{a}$ Departamento de Física, Facultad de Ciencias Exactas y Naturales, Universidad de Buenos Aires, \\ Cuidad Universitaria, Buenos Aires 1428, Argentina \\ ${ }^{b}$ Departamento de Ciências Exatas e Tecnológicas, Universidade Estadual de Santa Cruz, \\ Rodov. Jorge Amado km 16, CEP: 45.662-900, Ilhéus, BA, Brasil \\ ${ }^{c}$ Universidad de Buenos Aires, Facultad de Ciencias Exactas y Naturales, Departamento de Física, \\ Buenos Aires C1428EGA, Argentina \\ ${ }^{d}$ CONICET-Universidad de Buenos Aires, Facultad de Ciencias Exactas y Naturales, \\ Instituto de Física de Buenos Aires (IFIBA), \\ Buenos Aires C1428EGA, Argentina \\ E-mail: nahuelmg@df.uba.ar, kandus@uesc.br, calzetta@df.uba.ar
}

ABSTRACT: In the Second Order Theories (SOT) of real relativistic fluids, the non-ideal properties of the flows are described by a new set of dynamical tensor variables. In this work we explore the non-linear dynamics of those variables in a conformal fluid. Among all possible SOTs, we choose to work with the Divergence Type Theories (DTT) formalism, which ensures that the second law of thermodynamics is fulfilled non-perturbatively. The tensor modes include two divergence-free modes which have no analog in theories based on covariant generalizations of the Navier-Stokes equation, and that are particularly relevant because they couple linearly to a gravitational field. To study the dynamics of this irreducible tensor sector, we observe that in causal theories such as DTTs, thermal fluctuations induce a stochastic stirring force, which excites the tensor modes while preserving energy momentum conservation. From fluctuation-dissipation considerations it follows that the random force is Gaussian with a white spectrum. The irreducible tensor modes in turn excite vector modes, which back-react on the tensor sector, thus producing a consistent non-linear, second order description of the divergence-free tensor dynamics. Using the Martin-Siggia-Rose (MSR) formalism plus the Two-Particle Irreducible Effective Action (2PIEA) formalism, we obtain the one-loop corrected equations for the relevant two-point correlation functions of the model: the retarded propagator and the Hadamard function. The overall result of the self-consistent dynamics of the irreducible tensor modes at this order is a depletion of the spectrum in the UV sector, which suggests that tensor modes could sustain an inverse entropy cascade.

KEYwords: Conformal Field Theory, Quark-Gluon Plasma

ARXiv EPRINT: 2002.08323 


\section{Contents}

1 Introduction 1

2 The model 5

2.1 Landau-Lifshitz hydrodynamics in a nut-shell 6

2.2 Minimal conformal DTT beyond Landau-Lifshitz hydrodynamics 8

$\begin{array}{ll}2.3 \text { Propagation speeds and "incompressibility" } & 12\end{array}$

3 The Martin-Siggia-Rose effective action $\quad 12$

3.1 Fluctuation-dissipation theorem in a DTT framework 13

$\begin{array}{lll}3.2 & \text { MSR and the 2PI effective action } & 15\end{array}$

$\begin{array}{lll}3.3 & \text { Induced dynamics } & 17\end{array}$

$\begin{array}{llr}4 & \text { Linear fluctuations around equilibrium } & 18\end{array}$

$\begin{array}{lll}4.1 & \text { Identifying the physical degrees of freedom } & 18\end{array}$

$\begin{array}{ll}4.2 \text { The quadratic action } & 21\end{array}$

4.3 The lowest order propagators 21

$\begin{array}{lll}\text { 4.3.1 Causal vector correlations } & 21\end{array}$

$\begin{array}{lll}\text { 4.3.2 Causal tensor correlations } & 22\end{array}$

$\begin{array}{lll}\text { 4.3.3 Hadamard vector correlations } & 23\end{array}$

4.3.4 Hadamard tensor correlations 24

5 Nonlinear fluctuations around equilibrium $\quad \mathbf{2 5}$

5.1 Cubic terms in the "classical" action 25

5.2 Tensor self-energy 26

$\begin{array}{lll}5.3 & \text { Tensor noise-kernel } & 30\end{array}$

$\begin{array}{lll}5.4 & \text { Nonlinear tensor correlations } & 31\end{array}$

5.5 Entropy and EMT fluctuations 31

6 Conclusions and discussion $\quad 32$

A Scaling of the relevant diagrams 33

\section{Introduction}

Fluid description of relativistic matter proved to be a powerful tool for a clearer understanding of high energy phenomena [1,2]. Examples are the thermalization [3] and isotropization [4] of the quark-gluon plasma created in the Relativistic Heavy Ion Collider (RHIC) facilities, the behaviour of matter in the inner cores of Neutron Stars (NS) [5-7] and during cosmological phase transitions [8], etc. The processes observed in those systems cannot be explained using ideal relativistic fluids. 
Unlike non-relativistic hydrodynamics, where there is a successful theory to describe non-ideal fluids, namely the Navier-Stokes equation, there is no definite mathematical model to study real relativistic fluids. The history of the development of such theory begins with the recognition of the parabolic character of Navier-Stokes and Fourier equations ${ }^{1}$ [9], which implies that they cannot be naively extended to relativistic regimes. In fact, the first attempts by Eckart and Landau $[10,11]$ to build a relativistic theory of dissipative fluids starting from the non-relativistic formulation, also encountered this pathology.

The paradox about the non-causal structure of Navier-Stokes and Fourier equations, known as First Order Theories (FOTs), was resolved phenomenologically in 1967 by I. Müller [12]. He showed that by including second order terms in the heat flow and the stresses in the conventional expression for the entropy, it was possible to obtain a system of phenomenological equations which was consistent with the linearized form of Grad kinetic equations [13], i.e., equations that describe transient effects that propagate with finite velocities. These equations, constitute the so-called Second Order Theories (SOTs), whose main difference with respect to FOTs is that the stresses are upgraded to dynamical variables that satisfy a set of Maxwell-Cattaneo equations [14-17], thus constituting a hyperbolic theory. Later on, Müller's phenomenological theory was extended to the relativistic regime by W. Israel and J.M. Stewart, and others [18-31].

The problem with parabolic evolution equations is not restricted to relativistic fluids. Hyperbolic theories are useful not only in the relativistic domain, but also in the nonrelativistic one, whenever the relaxation time toward a steady state is larger than the time between collisions as, e.g., second sound in superfluids and solids, polymeric fluids, etc. $[32,33]$. The difference between parabolic and hyperbolic theories can be ignored only when the relaxation times are shorter than the characteristic time scale of the state. This was originally suggested by Maxwell [14]. Muronga [34] compared the descriptions of early stages in heavy ion collisions given by ideal, FOTs and SOTs, and concluded that the latter were the most accurate and reliable among them.

The rationale for pursuing second order theories such as DTTs or anisotropic hydrodynamics [4] also comes from the observation that highly anisotropic expansions such as in the early stages of RHICs, produce strong local momentum anisotropies that cannot be described considering weak deviations of the distribution function from local equilibrium. Also, hydrodynamic formalisms derived from Grad's theory are liable to predict spurious instabilities not present in the underlying kinetic theory, for example, when considering perturbations of highly anisotropic flows [35], while SOTs give a more accurate description. As a matter of fact, not all SOTs are able to coup with highly anisotropic states: even for moderate specific shear viscosities $\eta / s \sim 5-10$ the (negative) longitudinal component of the viscous shear pressure can become so large in Israel-Stewart theory, that it overwhelms the thermal pressure, resulting in a negative total pressure along the beam direction [36, 37].

In 1996 Liu, Müller and Ruggieri [38] developed a field-like description of particle density, particle flux and energy-momentum components. The resulting field equations

\footnotetext{
${ }^{1}$ Recall that the non-relativistic Fourier law allows for an instantaneous propagation of heat.
} 
were the conservation of particle number, energy momentum and balance of fluxes, and were strongly constrained by the relativity principle, the requirement of hyperbolicity and the entropy principle. The only unknown functions of the formalism were the shear and bulk viscosities and the heat conductivity, and all propagation speeds were finite. Several years later, Geroch and Lindblom extended the analysis of Liu et al. and wrote down a general theory were all the dynamical equations can be written as total-divergence equations [39, 40], see also refs. [35, 41-47]. This theory, known as Divergence Type Theory (DTT) is causal in an open set of states around equilibrium states. Moreover, all the dynamics is determined by a single scalar generating functional of the dynamical variables, a fact that allows to cast the theory in a simple mathematical form. Besides the dynamical equations an extra four-vector current is introduced, the entropy four-current, which is a function of the basic fields and not of any of their derivatives, and whose divergence is non-negative by the sole virtue of the dynamical equations. This fact guarantees that the second law is automatically enforced at all orders in a perturbative development. In contrast, as Israel-Stewart-like theories must be built order by order, the Second Law of thermodynamics must be enforced in each step of the construction [48]. In other words, DTTs are exact hydrodynamic theories that do not rely on velocity gradient expansions and therefore go beyond Israel-Stewart-like second-order theories. Further, DTTs have been tested through its application to Bjorken and Gubser flows [47], where exact solutions of the kinetic theory are available.

The novelty of SOTs, is the introduction of tensor dynamical variables to account for non-ideal features of the flow. This means that besides the scalar (spin 0 ) and vector (spin 1) modes already present in Landau-Lifshitz or Eckart theories, it is possible to excite tensor (spin 2) perturbations. This fact enlarges the set of hydrodynamic effects that a real relativistic fluid can sustain. As is familiar for the gravitational field, the tensor sector can be further decomposed into scalar, vector, and divergence-free components. If present in the Early Universe plasma, the latter could excite primordial gravitational waves [49], or seed primordial electromagnetic fluctuations [50]. Another scenario where tensor modes could play a relevant role are high energy astrophysical compact objects as, e.g., Neutron Stars [6]. It is well known that rotational tensor normal modes of those stars can source gravitational waves, however at present there is no compelling hydrodynamical model for them.

The study of hydrodynamic fluctuations in the relativistic regime is a natural follow-up to the successful application of the theory to describe thermalization and isotropization in systems such as RHICs [3]. Among all the possible lines to pursue this task, there is the study of the stochastic flows induced by the fluid's own thermal fluctuations.

The development of fluctuating hydrodynamics was pioneered by Landau and Lifshitz [11, 51-54], who applied the fluctuation dissipation theorem [55] to the Navier-Stokes equation. Hydrodynamic fluctuations in a consistent relativistic theory were studied in [28] and, in the context of DTTs, in [45]. Forcing by thermal noise is relevant to the calculation of the transport coefficients of the fluid [56-58] as well as the phenomenon of long time tails [59-65]. Noise, whether thermal or not, can also play an important role in early Universe phenomena such as primordial magnetic field induction [66, 67] and phase transitions $[68,69]$ to cite a few. 
In the literature on relativistic fluctuating hydrodynamics, no distinctions were made between scalar and vector fluctuations, not even tensor fluctuations were mentioned. So, as the distinctive feature of SOTs is that they sustain tensor modes, in this work we shall concentrate on the study of fluctuations in the pure tensor sector. Among all possible SOTs, we choose to work with DTTs. The reasons were mentioned above: they are thermodynamically and relativistically consistent in arbitrary flows and independently of any approximations. Consequently in a perturbative development we do not have to worry about enforcing the Second Law.

To address the subjet of study, we use effective field theory methods, which long ago began to be used in the study of turbulence [70-72], and continue to be a powerful tool to study random flows [73-76]. Among all methods, the Effective Action formalism allows to express the different $\mathrm{N}$-point correlation functions of the theory in terms of loop diagrams, which adds a new source of intuition in the intepretation of the correlations. In this manuscript we use the Two-Particle Irreducible Effective Action (2PIEA) formalism, through which we write down the evolution equations for the relevant two-point functions of the problem under study, namely the Retarded and the Hadamard propagators. This field method allows to build the propagators as the contribution of all closed interacting diagrams that cannot be separated by cutting two of their internal lines.

The Martin-Siggia-Rose (MSR) formalism [77-82] is a systematic way to derive the sought 2PIEA, which then may be straightforwardly evaluated through a diagrammatic expansion [82-86]. These methods have been applied to relativistic fluids in refs. [3, 87-89]. To characterize the thermal fluctuations we shall use a formulation of the fluctuationdissipation theorem appropriate to causal hydrodynamics [45], recovering the LandauLifshitz fluctuating hydrodynamics as a limiting case.

In summary, the relativistic perfect fluid approach that is widely used in several high energy descriptions is rather well established [90], while relativistic viscous hydrodynamics is much less well understood. We are dealing with systems whose fundamental description, whether kinetic theory, field theory or a combination of both, involves many more degrees of freedom than hydrodynamics may capture. As most excitations decay exponentially fast, long lived modes can be considered an attractor in the space of solutions [91-94] regardless of the initial conditions. In this sense stochastic SOTs represent phenomenological attempts to model the longest-lived non-hydrodynamic modes as additional degrees of freedom (besides the usual hydrodynamic ones) and the short-lived as noise, in timescales of the order or shorter than the relaxation time, using causality as a guideline. Among all SOTs, DTTs have the advantage of being thermodynamically consistent. It is important to mention that recently there appeared several works indicating that causality might be satisfied within FOTs [95-97] (see also [98]). However our interest in SOTs is precisely the fact that its range of variables is broader, including specially divergenceless tensor modes which enable a richer description of irreversible processes. Further, it is well-known that the transverse and traceless projection of the anisotropic stress tensor, $\Pi_{\mathrm{TT}}^{i j}$, plays an important role in the dynamics of the gravitational waves acting as a source [99]. In the cosmological context there are several scenarios in which matter has non-vanishing $\Pi_{\mathrm{TT}}^{i j}$ and it affects the amplitude of gravitational waves [100], e.g. freely streaming neutrinos or photons, interacting scalar fields and primordial density perturbations, among others. 
The paper is organized as follows. In section 2 we begin by quickly reviewing the Landau-Lifshitz hydrodynamics, after which we build the minimal conformal DivergenceType-Theory beyond LL. We end this section by setting the criterion for incompressibility. In section 3 we give an abridged presentation of the fluctuation-dissipation theorem in DTTs consistent with a causal theory [45] and outline the Martin-Siggia-Rose formalism for the Two-Particle-Irreducible Effective Action. We briefly show how this formalism allows to write down evolution equations for the main propagators of the theory, namely the retarded (or causal) propagator and the Hadamard two-point function. In section 4 we study the effect of linear fluctuations around an equilibrium state and find the lowest order causal propagators as well as the vector and tensor Hadamard propagators. In section 5 we extended the analysis of section 4 to include nonlinear fluctuations. We work within the free-streaming approximation, which amounts to consider times shorter than the typical macroscopic relaxation time. Even at these very early times one may formulate a consistent hydrodynamics that acts as an attractor for the evolution of the system [92-94]. The free-streaming approximation is equivalent to the approximation $\tau \rightarrow$ $\infty$ which was recently shown to be appropriate for the early stages in high-energy heavy ion collisions [101]. In this regime we find that at one-loop in the interactions and using dimensional regularization to treat the ultraviolet divergences, the equation for the causal tensor propagator acquires a new term which renormalizes the relaxation time introducing a scale dependence proportional to the fourth power of the momentum. In consequence the Hadamard correlation function is also modified in a way that shows a transition from a flat spectrum, for low values of $p$, to a power law spectrum $\left(\propto p^{-4}\right)$ with increasing $p$.

Before going on a comment is in order. In the large- $\tau$ limit we are considering there may be several so-called non-hydrodynamical modes contributing to the dynamics. We have chosen to include only a single tensor mode as representative, because such a mode is naturally present in the energy-momentum tensor, and so it is easily identifiable in a large range of models. However, in the absence of a symmetry principle that singles out this tensor mode above other possible slow modes, such as higher spin currents, these extra modes may contribute similarly to loop corrections. In such a case, a more complex theory must be considered and the analysis presented in this paper is a preliminary first step in this direction. We end section 5 by briefly discussing the effect of the tensor fluctuations on the mean value of the entropy, which is also depleted in the "large- $p$ range" of the spectrum, arguing that this may be an indication of an inverse cascade of entropy [102]. Finally in section 6 we discuss the results we obtained, draw our main conclusions and suggest possible lines to pursue the study developed in this manuscript. We left for the appendix A the discussion of the scaling law of the main diagrams that contribute to the one-loop approximation.

\section{The model}

To make this manuscript self-contained, we begin this section with a condensed review of Landau-Lifshitz hydrodynamics for a conformal neutral fluid, in order to show that a FOT does not guarantee fulfillment of the Second Law of thermodynamics. Among all possible 
SOTs, we choose a DTT to build what is arguably the minimal extension of Landau-Lifshitz hydrodynamics which enforces the Second Law of thermodynamics non-perturbatively, and where the dynamics of the neutral fluid is given by the conservation laws of the energymomentum tensor (EMT) $T^{\mu \nu}$ and of a third order tensor $A^{\mu \nu \rho}$ that encodes the non-ideal properties of the flow. The theory is completed by considering an entropy current $S^{\mu}$ whose conservation equation enforces the Second Law of thermodynamics. $T^{\mu \nu}$ is symmetric and traceless, and $A^{\mu \nu \rho}$ is totally symmetric and traceless on any two indices [103]. We linearize the evolution equations and find the propagation speed for the scalar, vector and tensor modes, from which we write down a criterion to define incompressibility.

\subsection{Landau-Lifshitz hydrodynamics in a nut-shell}

Let us consider the simplest model for a conformal fluid, for which there is no particle number current and the energy-momentum tensor is traceless. The energy density $\rho$ is defined by the Landau prescription

$$
T^{\mu \nu} u_{\nu}=-\rho u^{\mu}
$$

with normalization $u^{2}=-1$. Observe that eq. (2.1) is also the definition of $u^{\mu}$ as an eigenvector of $T^{\mu \nu}$ with eigenvalue $-\rho$. For an ideal fluid the energy momentum tensor must be isotropic in the rest frame, so

$$
T_{0}^{\mu \nu}=\rho u^{\mu} u^{\nu}+p \Delta^{\mu \nu}
$$

where

$$
\Delta^{\mu \nu}=g^{\mu \nu}+u^{\mu} u^{\nu}
$$

is the projector onto hypersurfaces orthogonal to $u^{\mu}$. Tracelessness implies the equation of state

$$
p=\frac{\rho}{3} .
$$

From the entropy density $s=(\rho+p) / T_{L L}$ we build the entropy flux

$$
S_{0}^{\mu}=s u^{\mu}=p \beta_{L L}^{\mu}-\beta_{L L \nu} T_{0}^{\mu \nu}
$$

with $\beta_{L L}^{\mu}=u^{\mu} / T_{L L}$ and where subindex $L L$ refers to Landau-Lifshitz frame. The differential form for the first law, $d s=d \rho / T_{L L}$, implies

$$
d S_{0}^{\mu}=-\beta_{L L \nu} d T_{0}^{\mu \nu}
$$

which gives that an ideal fluid flows with no entropy production, i.e.,

$$
S_{0 ; \mu}^{\mu}=-\beta_{L L \nu} T_{0 ; \mu}^{\mu \nu}=0 .
$$

Besides from $p=\rho / 3$ we have $s=4 \rho / 3 T_{L L}$ and $d s / d \rho=1 / T_{L L}$ and we get $\rho=\sigma T_{L L}^{4}$, where $\sigma$ is a dimensionless constant which depends on the statistics and the number of species of the particles that describe the fluid. In the case of a photon gas $\sigma$ is the wellknown Stefan-Boltzmann constant. 
A real fluid departs from an ideal one in that now

$$
T^{\mu \nu}=T_{0}^{\mu \nu}+\Pi^{\mu \nu}
$$

where $\Pi^{\mu \nu}$ encodes the non-ideal properties of the flow and satisfies $\Pi^{\mu \nu} u_{\nu}=0$. If we still consider $S_{0}^{\mu}$ to be the entropy flux, we now have

$$
S_{0 ; \mu}^{\mu}=-\beta_{L L \nu} T_{0 ; \mu}^{\mu \nu}=\beta_{L L \nu} \Pi_{; \mu}^{\mu \nu}=-\frac{u_{\nu ; \mu}}{T_{L L}} \Pi^{\mu \nu} .
$$

Positive entropy production is satisfied if

$$
\Pi_{\mu \nu}=-\eta \sigma_{\mu \nu}
$$

where $\sigma^{\mu \nu}$ is the shear tensor

$$
\sigma^{\mu \nu}=\Delta^{\mu \sigma} \Delta^{\nu \lambda}\left(u_{\sigma ; \lambda}+u_{\lambda ; \sigma}-\frac{2}{3} \Delta_{\sigma \lambda} u_{; \rho}^{\rho}\right)
$$

and $\eta \propto T_{L L}^{3}$ is the fluid viscosity. This constitutive relation leads to Landau-Lifshitz hydrodynamics, namely a covariant Navier-Stokes equation, which violates causality [23].

We may intend to solve the problem by upgrading $\Pi^{\mu \nu}$ to a dynamical variable and adopting a Maxwell-Cattaneo equation for it, having eq. (2.10) as an asymptotic limit. We then write

$$
\Pi_{\mu \nu}=-\left[\eta \sigma_{\mu \nu}+\tau \dot{\Pi}^{\mu \nu}\right] .
$$

This would follow from demanding positive entropy production with an entropy production term

$$
S_{; \mu}^{\mu}=-\frac{\Pi^{\mu \nu}}{2 T_{L L}}\left[\sigma_{\mu \nu}+\varsigma \dot{\Pi}_{\mu \nu}\right]
$$

and identifying later on $\tau=\varsigma \eta$. There arises the problem of what is $S^{\mu}$. A natural choice would be

$$
S^{\mu}=S_{0}^{\mu}-\frac{\varsigma}{4 T_{L L}} u^{\mu} \Pi^{\gamma \nu} \Pi_{\gamma \nu}
$$

which is thermodynamically satisfactory, but leads to

$$
S_{; \mu}^{\mu}=-\frac{\Pi^{\mu \nu}}{2 T_{L L}}\left[\sigma_{\mu \nu}+\varsigma \dot{\Pi}_{\mu \nu}\right]-\frac{\varsigma}{4} \Pi^{\gamma \nu} \Pi_{\gamma \nu} \beta_{L L ; \mu}^{\mu} .
$$

The extra term may be expected to be small, as it is of third order in deviations from equilibrium, but it is not nonnegative definite, and so we cannot be certain that the Second Law is properly enforced. To guarantee that it is, we should go to higher order in eq. (2.12), a step that would stem from including a new higher order term in the expression (2.13), and then impose a condition equivalent to (2.14), and so on. In other words, in order to have a thermodynamically consistent hydrodynamics we should enforce the Second Law order by order in deviations from equilibrium. 


\subsection{Minimal conformal DTT beyond Landau-Lifshitz hydrodynamics}

Instead of patching the theory one step at a time, DTTs attempt to formulate a consistent theory in its own right by postulating new currents, besides $T^{\mu \nu}$, which together determine the entropy flux. In its simplest form there is only one further current, $A^{\mu \nu \rho}$, satisfying a divergence-type equation [103]

$$
A_{; \rho}^{\mu \nu \rho}=I^{\mu \nu}
$$

where $I^{\mu \nu}$ is a tensor source of irreversibility. A simple count of degrees of freedom tells us that we need 5 independent equations to complement the 4 equations from the energy momentum conservation. We impose $A^{\mu \nu \rho}$ to be totally symmetric and traceless on any two indices and take the transverse, traceless part of eq. (2.16) as providing the required equations.

The big assumption of DTTs is that we have a local First Law of the form

$$
d S^{\mu}=-\beta_{\nu} d T^{\mu \nu}-\zeta_{\nu \rho} d A^{\mu \nu \rho}
$$

with $\zeta_{\mu \nu}$ a new tensor variable that encodes the non-ideal properties of the flow. In particular, this leads to

$$
S_{; \mu}^{\mu}=-\zeta_{\nu \rho} I^{\mu \nu}
$$

So the Second Law is enforced as long as

$$
\zeta_{\mu \nu} I^{\mu \nu} \leq 0
$$

Another consequence of eq. (2.17) is that if we consider the Massieu function density [39, 40]

$$
\Phi^{\mu}=S^{\mu}-\beta_{\nu} T^{\mu \nu}-\zeta_{\nu \rho} A^{\mu \nu \rho}
$$

Then

$$
\begin{aligned}
& \frac{\partial \Phi^{\mu}}{\partial \beta_{\nu}}=T^{\mu \nu} \\
& \frac{\partial \Phi^{\mu}}{\partial \zeta_{\nu \rho}}=A^{\mu \nu \rho}
\end{aligned}
$$

and further, the symmetry of $T^{\mu \nu}$ allows us to write

$$
\Phi^{\mu}=\frac{\partial \Phi}{\partial \beta_{\mu}}
$$

Thus the theory is defined by specifying the scalar $\Phi$ and the tensor $I^{\mu \nu}$ as local functions of the vector $\beta^{\mu}$ and the tensor $\zeta^{\mu \nu}$, subjected to eq. (2.19). Although a DTT may be derived from an underlying microscopic description, such as kinetic or field theory, when such is available, one of its appealing features is that one can go a long way into finding the right DTT from purely macroscopic arguments. In this paper we shall make the simplifying assumption that the scalar $\Phi$ has no space-time dependence other than through the hydrodynamical variables themselves, for more general situations see [47]. 
We start by writing the generating function $\Phi$ in terms of 0 th, 1st and 2nd order in deviations from equilibrium

$$
\Phi=\Phi_{0}+\Phi_{1}+\Phi_{2}
$$

In equilibrium $\zeta^{\mu \nu}=0$, so

$$
\Phi_{0}=\phi_{0}(X)
$$

where $X=\beta_{\mu} \beta^{\mu}$. Thus

$$
\begin{gathered}
\Phi_{0}^{\mu}=2 \beta^{\mu} \phi_{0}^{\prime}(X) \\
T_{0}^{\mu \nu}=4 \beta^{\mu} \beta^{\nu} \phi_{0}^{\prime \prime}(X)+2 g^{\mu \nu} \phi_{0}^{\prime}(X)
\end{gathered}
$$

then

$$
0=T_{\mu}^{\mu}=4\left[X \phi_{0}^{\prime \prime}+2 \phi_{0}^{\prime}\right]
$$

Thus the only choice is

$$
\begin{aligned}
\phi_{0} & =-\frac{\sigma}{6} X^{-1} \\
\Phi_{0}^{\mu} & =\frac{\sigma}{3} \beta^{\mu} X^{-2} \\
T_{0}^{\mu \nu} & =\sigma X^{-2}\left[-X^{-1} \beta^{\mu} \beta^{\nu}+\frac{1}{3} \Delta^{\mu \nu}\right]
\end{aligned}
$$

For the first order terms we have

$$
\begin{aligned}
\Phi_{1} & =\phi_{1}(X) \zeta_{\lambda}^{\lambda}+\phi_{2}(X) \zeta_{\lambda \sigma} \beta^{\lambda} \beta^{\sigma} \\
\Phi_{1}^{\mu} & =2 \beta^{\mu}\left[\phi_{1}^{\prime}(X) \zeta_{\lambda}^{\lambda}+\phi_{2}^{\prime}(X) \zeta_{\lambda \sigma} \beta^{\lambda} \beta^{\sigma}\right]+2 \phi_{2}(X) \zeta_{\sigma}^{\mu} \beta^{\sigma}
\end{aligned}
$$

whereby

$$
\begin{aligned}
T_{1}^{\mu \nu}= & 4 \beta^{\mu} \beta^{\nu}\left[\phi_{1}^{\prime \prime}(X) \zeta_{\lambda}^{\lambda}+\phi_{2}^{\prime \prime}(X) \zeta_{\lambda \sigma} \beta^{\lambda} \beta^{\sigma}\right]+2 g^{\mu \nu}\left[\phi_{1}^{\prime}(X) \zeta_{\lambda}^{\lambda}+\phi_{2}^{\prime}(X) \zeta_{\lambda \sigma} \beta^{\lambda} \beta^{\sigma}\right] \\
& +4 \phi_{2}^{\prime}(X)\left(\zeta_{\sigma}^{\mu} \beta^{\nu}+\zeta_{\sigma}^{\nu} \beta^{\mu}\right) \beta^{\sigma}+2 \phi_{2}(X) \zeta^{\mu \nu}
\end{aligned}
$$

and

$$
A_{1}^{\mu \nu \rho}=2 \beta^{\mu}\left[\phi_{1}^{\prime}(X) g^{\nu \rho}+\phi_{2}^{\prime}(X) \beta^{\nu} \beta^{\rho}\right]+\phi_{2}(X)\left(g^{\mu \nu} \beta^{\rho}+g^{\mu \rho} \beta^{\nu}\right)
$$

To obtain the right number of degrees of freedom for a conformal fluid we ask that for physically meaningful fields $\zeta_{\lambda}^{\lambda}=\zeta_{\lambda \sigma} \beta^{\sigma}=0$. When these conditions hold we shall say we are "on shell". As $A^{\mu \nu \rho}$ must be symmetric on any pair of indices we have that $\phi_{2}=2 \phi_{1}^{\prime}$, and by demanding $A^{\mu \nu \rho}$ to be traceless on any pair of indices $X \phi_{2}^{\prime}+3 \phi_{2}=0$. So

$$
\phi_{2}=-\frac{a}{2} X^{-3}
$$

with $a$ a constant that sets the intensity of the first order deviations around equilibrium, whose precise value will depend on the specific system under study, and

$$
\begin{aligned}
\Phi_{1}^{\mu} & =0 \\
T_{1}^{\mu \nu} & =-a X^{-3} \zeta^{\mu \nu} \\
A_{1}^{\mu \nu \rho} & =3 a X^{-4} \beta^{\mu} \beta^{\nu} \beta^{\rho}-\frac{1}{2} a X^{-3}\left(g^{\mu \nu} \beta^{\rho}+g^{\mu \rho} \beta^{\nu}+g^{\nu \rho} \beta^{\mu}\right)
\end{aligned}
$$


In writing second order terms, we leave out terms that do not contribute to the currents "on shell". This leaves

$$
\Phi_{2}=\phi_{3}(X) \zeta_{\lambda}^{2 \lambda}+\phi_{4}(X) \zeta_{\lambda \sigma}^{2} \beta^{\lambda} \beta^{\sigma}
$$

and

$$
\Phi_{2}^{\mu}=2 \phi_{3}^{\prime}(X) \zeta_{\lambda}^{2 \lambda} \beta^{\mu}+2 \phi_{4}^{\prime}(X) \zeta_{\lambda \sigma}^{2} \beta^{\lambda} \beta^{\sigma} \beta^{\mu}+\phi_{4}(X) \zeta_{\lambda \sigma}^{2}\left[g^{\lambda \mu} \beta^{\sigma}+g^{\sigma \mu} \beta^{\lambda}\right]
$$

Therefore

$$
T_{2}^{\mu \nu}=4 \phi_{3}^{\prime \prime}(X) \zeta_{\lambda}^{2 \lambda} \beta^{\mu} \beta^{\nu}+2 \phi_{3}^{\prime}(X) \zeta_{\lambda}^{2 \lambda} g^{\mu \nu}+\phi_{4}(X) \zeta_{\lambda \sigma}^{2}\left[g^{\lambda \mu} g^{\sigma \nu}+g^{\lambda \nu} g^{\sigma \mu}\right]
$$

and

$$
A_{2}^{\mu \rho \sigma}=8 \phi_{3}^{\prime}(X) \zeta^{\rho \sigma} \beta^{\mu}+2 \phi_{4}(X)\left[\zeta^{\rho \mu} \beta^{\sigma}+\zeta^{\sigma \mu} \beta^{\rho}\right]
$$

From $T_{2 \mu}^{\mu}=0$ we get

$$
4 X \phi_{3}^{\prime \prime}+8 \phi_{3}^{\prime}+2 \phi_{4}=0
$$

From symmetry of $A^{\mu \nu \rho}$ we get $\phi_{4}=4 \phi_{3}^{\prime}$. So we can write

$$
\begin{aligned}
\Phi_{2}^{\mu} & =\frac{b}{4} X^{-4} \beta^{\mu} \zeta_{\lambda}^{2 \lambda} \\
T_{2}^{\mu \nu} & =b X^{-4}\left[\zeta^{2 \mu \nu}+\frac{1}{4}\left(g^{\mu \nu}-8 X^{-1} \beta^{\mu} \beta^{\nu}\right) \zeta_{\lambda}^{2 \lambda}\right] \\
A_{2}^{\mu \nu \rho} & =\frac{b}{2} X^{-4}\left(\zeta^{\mu \nu} \beta^{\rho}+\zeta^{\mu \rho} \beta^{\nu}+\zeta^{\nu \rho} \beta^{\mu}\right)
\end{aligned}
$$

with $b$ a constant that sets the amplitude of the second order deviations around equilibrium. Its precise value will depend on the specific system under study. The energy density then is

$$
\rho=-X^{-1} \beta_{\nu} \beta_{\mu} T^{\mu \nu}=X^{-2}\left[\sigma+\frac{7}{4} b X^{-2} \zeta_{\lambda}^{2 \lambda}\right]
$$

and the entropy current reads

$$
S^{\mu}=\frac{4}{3} \sigma \beta^{\mu} X^{-2}+\frac{3}{2} b X^{-4} \beta^{\mu} \zeta_{\lambda}^{2 \lambda} \approx \frac{4}{3} \rho \beta^{\mu}\left[1-\frac{5}{8} \frac{b}{\sigma} X^{-2} \zeta_{\lambda}^{2 \lambda}\right]
$$

We now define

$$
\begin{aligned}
\zeta^{\mu \nu} & =-\sqrt{\frac{\sigma}{b}} X Z^{\mu \nu} \\
a & =\alpha \sqrt{b \sigma}
\end{aligned}
$$

where $\alpha$ is to be defined below, to get

$$
\begin{aligned}
T^{\mu \nu}= & \sigma X^{-2}\left[-X^{-1} \beta^{\mu} \beta^{\nu}+\frac{1}{3} \Delta^{\mu \nu}+\alpha Z^{\mu \nu}+Z^{2 \mu \nu}\right. \\
& \left.+\frac{1}{4}\left(g^{\mu \nu}-8 X^{-1} \beta^{\mu} \beta^{\nu}\right) Z_{\lambda}^{2 \lambda}\right] \\
A^{\mu \nu \rho}= & \frac{1}{2} a X^{-4}\left\{6 \beta^{\mu} \beta^{\nu} \beta^{\rho}-X\left[g^{\mu \nu} \beta^{\rho}+g^{\mu \rho} \beta^{\nu}+g^{\nu \rho} \beta^{\mu}\right.\right. \\
& \left.\left.+\alpha^{-1}\left(Z^{\mu \nu} \beta^{\rho}+Z^{\mu \rho} \beta^{\nu}+Z^{\nu \rho} \beta^{\mu}\right)\right]\right\} \\
S^{\mu}= & \frac{4}{3} \sigma \beta^{\mu} X^{-2}\left[1+\frac{9}{8} Z_{\lambda}^{2 \lambda}\right]
\end{aligned}
$$


These constitutive relations define the theory. By comparing to the Landau-Lifshitz theory above we see that on dimensional grounds we may write $X^{-1}=-T^{2}$, where $T$ has dimensions of temperature, while $Z^{\mu \nu}$ is dimensionless. Writing $\beta^{\mu}=u^{\mu} / T$ the constitutive relations take the form

$$
\begin{aligned}
T^{\mu \nu}= & \sigma T^{4}\left[\left(1+\frac{7}{4} Z_{\lambda}^{2 \lambda}\right)\left(u^{\mu} u^{\nu}+\frac{1}{3} \Delta^{\mu \nu}\right)+\alpha Z^{\mu \nu}+\left(Z^{2 \mu \nu}-\frac{1}{3} \Delta^{\mu \nu} Z_{\lambda}^{2 \lambda}\right)\right] \\
A^{\mu \nu \rho}= & \frac{1}{2} a T^{5}\left\{6 u^{\mu} u^{\nu} u^{\rho}+\left[g^{\mu \nu} u^{\rho}+g^{\mu \rho} u^{\nu}+g^{\nu \rho} u^{\mu}\right.\right. \\
& \left.\left.+\alpha^{-1}\left(Z^{\mu \nu} u^{\rho}+Z^{\mu \rho} u^{\nu}+Z^{\nu \rho} u^{\mu}\right)\right]\right\} \\
S^{\mu}= & \frac{4}{3} \sigma u^{\mu} T^{3}\left[1+\frac{9}{8} Z_{\lambda}^{2 \lambda}\right]
\end{aligned}
$$

To fix the remaining constants we ask that the theory reproduces Landau-Lifshitz hydrodynamics to first order in deviations from equilibrium. This requires $T=T_{L L}\left(1+O\left(Z^{2}\right)\right)$ and

$$
\sigma \alpha T^{4} Z^{\mu \nu}=-\eta \sigma^{\mu \nu}
$$

In the DTT framework, eq. (2.57) ought to follow from the transverse, traceless part of the first-order conservation law for $A^{\mu \nu \rho}$

$$
I^{\mu \nu}=\Lambda_{\sigma \lambda}^{\mu \nu} A_{; \rho}^{\sigma \lambda \rho}=\frac{1}{2} a T^{5} \sigma^{\mu \nu}
$$

where

$$
\Lambda_{\sigma \lambda}^{\mu \nu}=\frac{1}{2}\left[\Delta_{\sigma}^{\mu} \Delta_{\lambda}^{\nu}+\Delta_{\lambda}^{\mu} \Delta_{\sigma}^{\nu}-\frac{2}{3} \Delta^{\mu \nu} \Delta_{\sigma \lambda}\right]
$$

is the complete transverse and traceless spatial projector. Therefore we must have

$$
I^{\mu \nu}=-\frac{a \sigma \alpha T^{6}}{2} \frac{T^{3}}{\eta} Z^{\mu \nu}
$$

It is convenient to introduce the relaxation time $\tau$ through the Anderson-Witting prescription [104, 105]

$$
I^{\mu \nu}=-\frac{a T^{5}}{2 \alpha \tau} Z^{\mu \nu}
$$

whereby

$$
\alpha^{2}=\frac{1}{\sigma T \tau}\left(\frac{\eta}{T^{3}}\right)
$$

We may estimate $\eta$ from the AdS/CFT bound [106], $\eta \geq(4 / 3) \sigma T_{0}^{3} / 4 \pi, T_{0}$ being a fiducial equilibrium temperature. In the next subsection we show that causality requires $\alpha^{2} \leq 2 / 3$ and so $T \tau \geq 3 \eta / 2 \sigma T_{0}^{3} \geq 1 / 2 \pi$. In what follows we shall be interested in the "freestreaming" limit $\tau \rightarrow \infty$, thus $\alpha \rightarrow 0$.

As we shall show in next section, when thermal fluctuations are considered $I^{\mu \nu}$ acquires a stochastic component, $I^{\mu \nu} \rightarrow I^{\mu \nu}+F^{\mu \nu}$. $F^{\mu \nu}$ is a stochastic source which may be derived from fluctuation-dissipation considerations and will be described in more detail below. Observe that this force sources entropy and not energy, as there is no stirring in the equations for the conservation of $T^{\mu \nu}$. 


\subsection{Propagation speeds and "incompressibility"}

Before we proceed, we shall derive the propagation speeds for different types of linearized fluctuations around equilibrium [107], and show that in the $\alpha \rightarrow 0$ limit scalar modes may be regarded as frozen, thus the fluid behaves as an incompressible one.

We are looking at a situation where a discontinuity is propagating along the surface $z=c t, c$ being the desired propagation speed. Above the front the fluid is in equilibrium, so $T=T_{0}=$ constant, $u^{\mu}=U^{\mu}=(1,0,0,0)$ and $Z^{\mu \nu}=0$. Hydrodynamic variables are continuous across the front. Observe that any variable $\mathcal{X}$ which remains constant at the front must obey $\dot{\mathcal{X}}=-c \mathcal{X}^{\prime}$, where $\mathcal{X}^{\prime}=\mathcal{X}_{3}$, where subindex 3 refers to the $z$ coordinate. Moreover the conditions $u^{2}=-1$ and $Z^{\mu \nu} u_{\nu}=0$ show that $u_{, \rho}^{0}=Z_{, \rho}^{\mu 0}=0$, and the condition $Z_{\rho}^{\rho}=0$ shows that $Z_{a}^{a}=-Z_{3}^{3}$ (indices $a, b$ run from 1 to 2 , which denote $x$ and $y$ coordinates).

The theory decomposes into tensor modes $Z^{a b}+(1 / 2) \delta^{a b} Z_{3}^{3}$, which do not propagate, vector modes $u^{a}$ and $Z^{a 3}$ and scalar modes $T, u^{3}$ and $Z^{33}$. Writing the conservation equations $T_{, \nu}^{\mu \nu}=0$ and $\Lambda_{\rho \lambda}^{\mu \nu} A_{, \sigma}^{\rho \lambda \sigma}=0$ (since $I^{\mu \nu}=0$ at the front), and eliminating time derivatives, we get two sets of equations. For the vector modes

$$
\begin{aligned}
u_{a}^{\prime}-\frac{c}{\alpha} Z_{a 3}^{\prime} & =0 \\
-\frac{4}{3} c u_{a}^{\prime}+\alpha Z_{a 3}^{\prime} & =0
\end{aligned}
$$

which shows that vector modes propagate with speed

$$
c_{V}^{2}=3 \alpha^{2} / 4
$$

For scalar modes we get

$$
\begin{aligned}
\frac{4}{3} c u_{3}^{\prime}-\frac{c}{\alpha} Z_{33}^{\prime} & =0 \\
\frac{T^{\prime}}{T_{0}}-c u_{3}^{\prime}+\frac{3}{4} \alpha Z_{33}^{\prime} & =0 \\
-c \frac{T^{\prime}}{T_{0}}+\frac{1}{3} u_{3}^{\prime} & =0
\end{aligned}
$$

which admits a non-propagating mode with $u_{3}^{\prime}=0, T^{\prime} / T_{0}=-(3 / 4) \alpha Z_{33}^{\prime}$, and two propagating modes with speed

$$
c_{S}^{2}=\frac{1}{3}+\alpha^{2}
$$

So causality demands $\alpha^{2} \leq 2 / 3$, and $c_{S} \gg c_{V}$ when $\alpha^{2} \ll 2 / 3$. This means that the only interaction of interest is between tensor and vector modes.

\section{The Martin-Siggia-Rose effective action}

As stated above, we shall be interested in fluids stirred by their own thermal fluctuations. Therefore in this section we shall review the fluctuation dissipation theorem appropriate to causal relativistic real fluids [45] (see also [28, 108]), and then use the MSR formulation [77-82] to develop an effective action from where we can get the Dyson equations for the stochastic correlations of vector and tensor hydrodynamic variables. 


\subsection{Fluctuation-dissipation theorem in a DTT framework}

In this subsection we summarize the derivation of the fluctuation-dissipation theorem as applied to causal relativistic fluid theories. Following ref. [45] we define the following shorthand notation for the variables described above

$$
\begin{aligned}
X^{A} & =\left(\beta^{a}, \zeta^{a b}\right), \\
A_{A}^{a} & =\left(T^{a b}, A^{a b c}\right), \\
I_{B} & =\left(0, I_{a b}\right) \\
F_{B} & =\left(0, F_{a b}\right), \\
S^{a} & =\Phi^{a}-X^{B} A_{B}^{a}, \\
A_{B}^{a} & =\frac{\delta \Phi^{a}}{\delta X^{B}},
\end{aligned}
$$

with $\Phi^{a}$ the vector generating functional. The entropy production is given by

$$
S_{; a}^{a}=-X^{B} A_{B ; a}^{a} .
$$

To include thermal fluctuations we add random sources $F_{B}$ in the equations of motion which then become Langevin-type equations, namely

$$
A_{B ; a}^{a}=I_{B}+F_{B}
$$

A satisfactory theory must predict vanishing mean entropy production in equilibrium, so

$$
\left\langle S_{; a}^{a}\right\rangle=-\left\langle X^{B}(x) I_{B}(x)\right\rangle-\left\langle X^{B}(x) F_{B}(x)\right\rangle=0 .
$$

However, because the coincidence limit may not be well defined, we impose a stronger condition due to elementary causality considerations, which is

$$
\left\langle X^{B}(x) I_{B}\left(x^{\prime}\right)\right\rangle+\left\langle X^{B}(x) F_{B}\left(x^{\prime}\right)\right\rangle=0
$$

for every space-like pair $\left(x, x^{\prime}\right)$. In the following we shall assume that we have defined the time in such a way that $x$ and $x^{\prime}$ belong to the same equal time surface, namely $t=t^{\prime}$. In the linear approximation $I_{B}$ is a linear function of $X^{C}$, then

$$
\left\langle X^{B}(x) I_{B}\left(x^{\prime}\right)\right\rangle=\int d^{3} x^{\prime \prime} I_{(B, C)}\left\langle X^{B}(x) X^{C}\left(x^{\prime \prime}\right)\right\rangle,
$$

with

$$
I_{(B, C)}=\frac{1}{2}\left[\frac{\delta I_{B}\left(x^{\prime}\right)}{\delta X^{C}\left(x^{\prime \prime}\right)}+\frac{\delta I_{C}\left(x^{\prime \prime}\right)}{\delta X^{B}\left(x^{\prime}\right)}\right] .
$$

Only the symmetrized derivative occurs in (3.11) due to the symmetry of the stochastic average. Assuming Gaussian white noise

$$
\left\langle F_{B}(x) F_{C}\left(x^{\prime}\right)\right\rangle=\sigma_{B C}\left(x, x^{\prime}\right) \delta\left(t-t^{\prime}\right),
$$

we have that the correlations between fluxes and noise is

$$
\left\langle X^{A}(x) F_{B}\left(x^{\prime}\right)\right\rangle=\int d^{3} x^{\prime \prime} \sigma_{B C}\left(x^{\prime}, x^{\prime \prime}\right) \frac{\delta X^{A}(x)}{\delta F_{C}\left(x^{\prime \prime}\right)},
$$


where $t^{\prime \prime}=t^{\prime}$. The fluctuation dissipation theorem follows from

$$
\left\langle X^{B}(x) X^{C}\left(x^{\prime}\right)\right\rangle=2 \frac{\delta X^{B}(x)}{\delta F_{C}\left(x^{\prime}\right)}=2 \frac{\delta X^{C}\left(x^{\prime}\right)}{\delta F_{B}(x)},
$$

whenever $t=t^{\prime}$, which implies

$$
\left\langle X^{B}(x) I_{B}\left(x^{\prime}\right)\right\rangle=\int d^{3} x^{\prime \prime} I_{(B, C)} \frac{\delta X^{B}(x)}{\delta F_{C}\left(x^{\prime \prime}\right)} .
$$

Using (3.10), (3.12), (3.14) and (3.16) we get

$$
\sigma_{B C}=-2 I_{(B, C)}
$$

or equivalently

$$
\left\langle F_{A}(x) F_{B}\left(x^{\prime}\right)\right\rangle=-\left[\frac{\delta I_{A}(x)}{\delta X^{B}\left(x^{\prime}\right)}+\frac{\delta I_{B}\left(x^{\prime}\right)}{\delta X^{A}(x)}\right] \delta\left(t-t^{\prime}\right) .
$$

We use this version of the fluctuation-dissipation theorem in order to set the correlation function of the noise source. Of course, as we show in the main text, in the limit in which our DTT converges to the Landau-Lifshitz hydrodynamics, the correlation of the stochastic energy-momentum tensor converges to the well-known Landau-Lifshitz noise [51, 52].

To verify eq. (3.15), let us multiply both sides by the non-singular matrix

$$
M_{A B}=n_{a} \frac{\delta^{2} \Phi^{a}}{\delta X^{A} \delta X^{B}}
$$

where $n_{a}$ is the unit normal field to the equal time surface containing both $x$ and $x^{\prime}$. In the linear approximation $\Phi^{a}$ is quadratic on $X^{A}$ and

$$
M_{A B} X^{B}=\frac{\delta\left(n_{a} \Phi^{a}\right)}{\delta X^{A}}=-\frac{\delta \Phi^{0}}{\delta X^{A}} .
$$

In equilibrium we may apply the Einstein's formula, relating the thermodynamic potentials to the distribution function of fluctuations, to conclude that

$$
M_{A B}\left\langle X^{B}(x) X^{C}\left(x^{\prime}\right)\right\rangle=-\delta_{A}^{C} \delta^{(3)}\left(x, x^{\prime}\right)
$$

where $\delta^{(3)}\left(x, x^{\prime}\right)$ is the three-dimensional covariant delta function on the Cauchy surface. This is a generalized version of the equipartition theorem. On the other hand

$$
M_{A B} \frac{\delta X^{B}(x)}{\delta F_{C}\left(x^{\prime}\right)}=-\frac{\delta A_{A}^{0}(x)}{\delta F_{C}\left(x^{\prime}\right)}
$$

with $A_{A}^{0}=-n_{a} A_{A}^{a}$. It is possible to write the equations of motion (3.8) as

$$
\frac{\partial A_{A}^{0}(x)}{\partial t}+L_{A}(x)=F_{A}(x)
$$

where $L_{A}$ involves the field variables on the surface, but not their normal derivatives, and $\partial / \partial t:=n^{a} \partial / \partial x^{a}$. Indeed

$$
\frac{\delta A_{A}^{0}(x)}{\delta F_{C}\left(x^{\prime}\right)}=\frac{1}{2} \delta_{A}^{C} \delta^{(3)}\left(x, x^{\prime}\right)
$$


The factor $1 / 2$ takes into account the average of the derivative evaluated in $x=x^{\prime-}$ and $x=x^{\prime+}$. Therefore (3.21) and (3.22) are equal. Due to the non-singularity of $M_{A B}$, equation (3.15) holds.

In the case at hand, these results imply that only the equation for $A^{\mu \nu \rho}$ acquires a random source, and then

$$
\left\langle F^{\mu \nu}(x) F_{\sigma \lambda}\left(x^{\prime}\right)\right\rangle=N \delta\left(t-t^{\prime}\right) \delta^{(3)}\left(x-x^{\prime}\right) \Lambda_{\sigma \lambda}^{\mu \nu}
$$

where

$$
N=\frac{a^{2} T_{0}^{7}}{\alpha^{2} \sigma \tau}
$$

\subsection{MSR and the 2PI effective action}

The main tools to study the evolution and physical properties of a stochastic system are their different propagators, or Green functions, because they determine the response of the system to its own thermal fluctuations as well as to the fluctuations in the initial conditions $[101,109,110]$. In the regime of strong fluctuations this study involves corrections due to non-linear effects [82-85] and it is in this scenario where effective field theory methods such as the MSR show their power [77-79, 81]; for applications to theories of turbulence see $[83,84,111]$. It was also used recently to study fluctuations in relativistic Landau-Lifshitz theory [87, 88].

We now proceed with the analysis of the correlations in the theory. From the analysis of the propagation velocities we know that in the free-streaming regime the scalar modes propagate faster than the vector ones and therefore can be considered as frozen. In other words, in the considered limit the flow may be regarded as "incompressible" (cfr. subsection 2.3). The MSR formalism will allow to convert the problem of classical fluctuations into a quantum field theory one, for which we shall derive the Two-Particle-Irreducible Effective Action (2PIEA). This formalism yields the Schwinger-Dyson equations for the propagators in the most direct way.

Before going on an important remark is in order. In hydrodynamics there is no explicit single 'small' parameter, such as $\hbar$ in quantum field theory, which can be used to organize the perturbative expansion. For this reason it has been propossed that the loop expansion should be understood as an expansion in 'the complexity of the interaction' [83, 84], since due to the randomness of the stirring, the sum of higher order terms will tend to cancel. On the other hand, it is possible to identify the relevant small parameters in the theory through the scaling behavior of restricted sets of graphs. In the case at hand, this analysis suggests that the loop expansion is an expansion in powers of $\left(p / p_{\mathrm{L}}\right)^{3}$ where

$$
p_{\mathrm{L}}=\left(c_{V}^{2} \sigma T_{0}^{3}\right)^{1 / 3} .
$$

(see appendix A). In consequence the loop expansion is consistent while $p<p_{\mathrm{L}}$.

Let us return to the construction of the 2PIEA. We continue to use the abridged notation from eqs. (3.1)-(3.6). The equations of motion have the form $P_{A}=F_{A}$, where the $P$ 's are the left hand sides of the EMT $T^{\mu \nu}$ and the nonequilibrium current $A^{\mu \nu \rho}$ conservation equations. If the sources $F_{A}$ are given, we call $X^{A}[F]$ the solution to the 
equations. Under thermal noise, all $\left\langle X^{A}\right\rangle=0$. Therefore we can write a generating functional for the correlation functions $\left\langle X^{A} X^{\beta}\right\rangle$

$$
e^{i W\left[K_{A B}\right]}=\int D X^{A} \int D F_{A} \mathcal{P}\left[F^{A}\right] e^{i \int K_{A B} X^{A} X^{B} / 2} \delta\left(X^{A}-X^{A}[F]\right)
$$

where $\mathcal{P}\left[F^{A}\right]$ is the Gaussian probability density for the sources, $K_{A B}$ are the currents introduced in the formalism to couple to the variables of the theory and the integration is performed over all the noise realizations. Observe that

$$
\delta\left(X^{A}-X^{A}[F]\right)=\operatorname{Det}\left[\frac{\delta P_{A}}{\delta X^{A}}\right] \delta\left(P_{A}-F_{A}\right)
$$

where the determinant can be proved to be a constant [112] and will be consequently disregarded. We exponentiate the delta function by adding auxiliary fields $Y_{A}$

$$
e^{i W\left[K_{A B}\right]}=\int D Y^{A} \int D X^{A} \int D F_{A} \mathcal{P}\left[F^{A}\right] e^{i \int K_{A B} X^{A} X^{B} / 2} e^{i \int Y^{A}\left(P_{A}-F_{A}\right)}
$$

Introducing the source correlations

$$
\left\langle F_{A} F_{B}\right\rangle=N_{A B}
$$

we finally obtain

$$
e^{i W\left[K_{A B}\right]}=\int D Y^{A} \int D X^{A} e^{i S} e^{i \int K_{A B} X^{A} X^{B} / 2}
$$

where

$$
S=\int d^{4} x\left[Y^{A} P_{A}+\frac{i}{2} Y^{A} N_{A B} Y^{B}\right]
$$

In fact, we have mapped the stochastic hydrodynamic problem into a nonequilibrium field theory one, where $S$ from eq. (3.33) plays the role of "classical" action. We may formally add new sources coupled to the auxiliary fields and consider the whole string $\mathcal{X}^{K}=\left(X^{A}, Y^{A}\right)$ as degrees of freedom of the theory.

The Legendre transform of the generating function is the 2PIEA $\Gamma\left[\mathcal{G}^{J K}\right]$, where the $\mathcal{G}^{J K}=\left\langle\mathcal{X}^{J} \mathcal{X}^{K}\right\rangle$ are the thermal correlations we seek. Once the 2PIEA is known, the actual correlations are obtained as extrema

$$
\frac{\delta \Gamma}{\delta \mathcal{G}^{J K}}=0
$$

The 2PIEA has the structure [113]

$$
\Gamma=\left.\frac{1}{2} \frac{\delta^{2} S}{\delta \mathcal{X}^{J} \delta \mathcal{X}^{J}}\right|_{\mathcal{X}=0} \mathcal{G}^{J K}-\frac{i}{2} \ln \operatorname{Det}\left[\mathcal{G}^{J K}\right]+\Gamma_{2 Q}
$$

where $\Gamma_{2 Q}$ is the sum of all two-particle irreducible Feynman graphs for a theory whose interactions are the terms cubic or higher in $S$, and carrying propagators $\mathcal{G}^{J K}$. 
It so happens that $\left\langle Y^{A} Y^{B}\right\rangle=0$, and also

$$
\left.\frac{\delta^{2} S}{\delta X^{A} \delta X^{B}}\right|_{\mathcal{X}=0}=\frac{\delta \Gamma_{2 Q}}{\delta\left\langle X^{A} X^{B}\right\rangle}=0
$$

so actually we get two sets of equations, one for the retarded propagators $G_{\mathrm{ret}}^{A A}=-i\left\langle X^{A} Y^{A}\right\rangle$

$$
\left\{\left.\frac{\delta^{2} S}{\delta Y^{B} \delta X^{A}}\right|_{\mathcal{X}=0}+2 \frac{\delta \Gamma_{2 Q}}{\delta\left\langle Y^{B} X^{A}\right\rangle}\right\} G_{\mathrm{ret}}^{A A}=\delta_{B}^{A}
$$

and another for the actual thermal correlations $G^{A B}=\left\langle X^{A} X^{B}\right\rangle$

$$
\left\{\left.\frac{\delta^{2} S}{\delta Y^{B} \delta X^{A}}\right|_{\mathcal{X}=0}+2 \frac{\delta \Gamma_{2 Q}}{\delta\left\langle Y^{B} X^{A}\right\rangle}\right\} G^{A B}+i\left\{\left.\frac{\delta^{2} S}{\delta Y^{B} \delta Y^{C}}\right|_{\mathcal{X}=0}+2 \frac{\delta \Gamma_{2 Q}}{\delta\left\langle Y^{B} Y^{C}\right\rangle}\right\} G_{\mathrm{adv}}^{C B}=0
$$

where $G_{\mathrm{adv}}^{C B}=-i\left\langle Y^{C} X^{B}\right\rangle$, with the integral

$$
G^{A B}=(-i) G_{\mathrm{ret}}^{A A} G_{\mathrm{adv}}^{B B}\left\{\left.\frac{\delta^{2} S}{\delta Y^{A} \delta Y^{B}}\right|_{\mathcal{X}=0}+2 \frac{\delta \Gamma_{2 Q}}{\delta\left\langle Y^{A} Y^{B}\right\rangle}\right\}
$$

\subsection{Induced dynamics}

We now begin to investigate the induced dynamic in the presence of thermal fluctuations. As we have seen, it is given by eqs. (3.37) for the causal, or retarded correlators and (3.38) for the symmetric, or Hadamard two-point functions. In equilibrium we have $\left\langle\mathcal{X}^{K}\right\rangle=0$. The MSR "classical" action (3.33) may be written as

$$
S_{C}\left[\mathcal{X}^{K}\right]=S_{Q}\left[\mathcal{X}^{K}\right]+S_{I}\left[\mathcal{X}^{K}\right]
$$

where $S_{Q}$ is quadratic and $S_{I}$ contains the interaction terms; in our case we only keep terms cubic in the fields in $S_{C}$. The 2PIEA is given by eq. (3.35), with

$$
e^{i \Gamma_{2 Q}}=\mathcal{N} \int D \mathcal{X}^{K} e^{-\frac{1}{2} \mathcal{X}^{K}\left(G^{-1}\right)_{K L} \mathcal{X}^{L}+i S_{C}\left[\mathcal{X}^{K}\right]}
$$

and where the $G^{K L}$ are the propagators

$$
G^{K L}=\left\langle\mathcal{X}^{K} \mathcal{X}^{L}\right\rangle
$$

and $\mathcal{N} \propto\left(\operatorname{Det} G^{K L}\right)^{-1 / 2}[113]$. We use the notation

$$
\langle\mathcal{O}\rangle=\mathcal{N} \int D \mathcal{X}^{K} e^{-\frac{1}{2} \mathcal{X}^{K}\left(G^{-1}\right)_{K L} \mathcal{X}^{L}} \mathcal{O}\left[\mathcal{X}^{K}\right]
$$

The normalization is set up so that $\langle 1\rangle=1$. If, as in our case, $\left\langle S_{C}\right\rangle=0$, then the lowest order contribution to $\Gamma_{2 Q}$ is

$$
\Gamma_{2 Q}=\frac{i}{2}\left\langle S_{C}^{2}\right\rangle
$$

and then the self energies read

$$
\Sigma_{K L}=2 \frac{\delta \Gamma_{2 Q}}{\delta G^{K L}}=i\left\langle\frac{\delta S_{C}}{\delta \mathcal{X}^{K}} \frac{\delta S_{C}}{\delta \mathcal{X}^{L}}\right\rangle
$$


The expectation value on the r.h.s. is developed in terms of Feynman graphs with propagators $G^{K L}$ in the internal legs, and where only 2PI graphs are considered [113]. Again to lowest order, we may replace the full propagators by their lowest order approximations

$$
\frac{\delta^{2} S_{Q}}{\delta \mathcal{X}^{K} \delta \mathcal{X}^{M}} G_{0}^{M L}=i \delta_{K}^{L}
$$

which describe the correlations of linearized fluctuations around equilibrium. The equations for nonlinear fluctuations (3.37) and (3.38) can then be written in compact form as

$$
\left[\frac{\delta^{2} S_{Q}}{\delta \mathcal{X}^{K} \delta \mathcal{X}^{M}}+\Sigma_{K M}\right] G^{M L}=i \delta_{K}^{L}
$$

\section{Linear fluctuations around equilibrium}

From the discussion above, to formulate a MSR effective action for the minimal DTT, we consider a "classical" action of the form (cfr. eq. (3.33))

$$
S=\frac{i}{2} N \int d^{4} x Y_{\mu \nu} Y^{\mu \nu}-\int d^{4} x\left\{Y_{\mu ; \nu} T^{\mu \nu}+Y_{\mu \nu ; \rho} A^{\mu \nu \rho}+Y_{\mu \nu} I^{\mu \nu}\right\}
$$

Since we are disregarding scalar modes, the auxiliary fields $Y_{\mu}$ and $Y_{\mu \nu}$ contain only vector and tensor degrees of freedom. This means that the independent variables are a three vector $Y^{j}$ and a three tensor $Y^{j k}$ obeying $Y_{; j}^{j}=Y_{; j}^{j k}=Y_{k}^{k}=0$. The time components are then constrained through $Y_{\mu} u^{\mu}=Y_{\mu \nu} u^{\mu}=Y_{\mu}^{\mu}=0$. Observe that $Y_{\mu}$ has units of $T^{-1}$, while $Y_{\mu \nu}$ has units of $T^{-2}$. Explicitly expr. (4.1) can be decomposed as $S=S_{N}+S_{T}+S_{A}+S_{I}$ with

$$
\begin{aligned}
S_{N}= & \frac{i N}{2} \int d^{4} x Y_{\mu \nu} Y^{\mu \nu} \\
S_{T}= & -\sigma T_{0}^{4} \int d^{4} x Y_{\mu ; \nu}\left[u^{\mu} u^{\nu}+\frac{1}{3} \Delta^{\mu \nu}+\alpha Z^{\mu \nu}+Z^{2 \mu \nu}+\frac{1}{4}\left(g^{\mu \nu}-8 u^{\mu} u^{\nu}\right) Z_{\lambda}^{2 \lambda}\right] \\
S_{A}= & -\frac{1}{2} a T_{0}^{5} \int d^{4} x Y_{\mu \nu ; \rho}\left[6 u^{\mu} u^{\nu} u^{\rho}+g^{\mu \nu} u^{\rho}+g^{\mu \rho} u^{\nu}+g^{\nu \rho} u^{\mu}\right. \\
& \left.+\frac{1}{\alpha}\left(Z^{\mu \nu} u^{\rho}+Z^{\mu \rho} u^{\nu}+Z^{\nu \rho} u^{\mu}\right)\right] \\
S_{I}= & \frac{T_{0}^{5}}{2 \alpha \tau} \int d^{4} x Y_{\mu \nu} Z^{\mu \nu}
\end{aligned}
$$

\subsection{Identifying the physical degrees of freedom}

We now apply the above formalism to study thermal fluctuations around a fiducial equilibrium configuration with velocity $U^{\mu}=(1,0,0,0)$ and temperature $T_{0}$. We shall keep only terms which are quadratic $\left(S_{q}\right)$ or cubic $S_{c}$, (not to be confused with the 'classical Action' referred above) in deviations from equilibrium. We shall derive the quadratic terms in the next subsection, and come back to the cubic terms later on, in subsection 5.1.

We write $u^{\mu}=U^{\mu}+V^{\mu}$. The condition $u^{2}=-1$ becomes $U_{\mu} V^{\mu}=-V^{2} / 2$ and so

$$
V^{\mu}=h_{\nu}^{\mu} V^{\nu}+\frac{1}{2} U^{\mu} V^{2}
$$


This suggests taking $V^{k}=\Delta_{\nu}^{k} V^{\nu}$ as independent variables, whereby

$$
V^{0}=\frac{1}{2}\left(V_{k} V^{k}-\left(V^{0}\right)^{2}\right) \approx \frac{1}{2} V_{k} V^{k}+\text { h.o. }
$$

Any transverse tensor admits a similar decomposition, namely

$$
\begin{aligned}
Y_{0} & \approx Y_{k} V^{k}+\text { h.o. } \\
Y_{0 j} & \approx Y_{k j} V^{k}+\text { h.o. } \\
Y_{00} & \approx Y_{k j} V^{k} V^{j}+\text { h.o. } \\
Z_{0 j} & \approx Z_{k j} V^{k}+\text { h.o. } \\
Z_{00} & \approx Z_{k j} V^{k} V^{j}+\text { h.o. }
\end{aligned}
$$

where h.o. means 'higher orders'. Moreover $Y_{k}^{k}=Z_{k}^{k}=0$. We can thus identify the quadratic and cubic terms in the "classical" action. The quadratic terms are

$$
\begin{aligned}
S_{N q} & =\frac{i N}{2} \int d^{4} x Y_{j k} Y^{j k} \\
S_{T q} & =-\sigma T_{0}^{4} \int d^{4} x\left[\frac{4}{3} Y_{j ; 0} V^{j}+\alpha Y_{j ; k} Z^{j k}\right] \\
S_{A q} & =-\frac{1}{2} a T_{0}^{5} \int d^{4} x\left[\frac{1}{\alpha} Y_{j k ; 0} Z^{j k}+Y_{j k ; l}\left(g^{j l} V^{k}+g^{k l} V^{j}\right)\right] \\
S_{I q} & =\frac{a T_{0}^{5}}{2 \alpha \tau} \int d^{4} x Y_{j k} Z^{j k}
\end{aligned}
$$

and recalling that $V_{; j}^{j}=Y_{; j}^{j}=Z_{; i j}^{i j}=Y_{; i j}^{i j}=0$, the cubic terms read

$$
\begin{aligned}
S_{T c}= & -\sigma T_{0}^{4} \int d^{4} x\left[-\alpha Y_{j ; 0} Z_{k j} V^{k}+Y_{j ; k}\left(\frac{4}{3} V^{j} V^{k}+Z^{j l} Z_{l}^{k}\right)\right] \\
S_{A c}= & -\frac{1}{2} a T_{0}^{5} \int d^{4} x\left[10\left(Y_{k j} V^{k}\right)_{; 0} V^{j}+\frac{2}{\alpha}\left(Y_{k j} V^{k}\right)_{; l} Z^{j l}\right. \\
& \left.+\frac{1}{\alpha} Y_{j k ; l}\left(Z^{j k} V^{l}+Z^{k l} V^{j}+Z^{l j} V^{k}\right)\right]
\end{aligned}
$$

Our next step is to Fourier transform all degrees of freedom. We adopt the convention

$$
V^{k}(\vec{x}, t)=\int \frac{d^{3} p}{(2 \pi)^{3}} e^{i \vec{p} \vec{x}} V^{k}(p, t)
$$

to get

$$
\begin{aligned}
S_{N q} & =\frac{i N}{2} \int d t \frac{d^{3} p}{(2 \pi)^{3}} Y_{j k}(-p, t) Y^{j k}(p, t) \\
S_{T q} & =-\sigma T_{0}^{4} \int d t \frac{d^{3} p}{(2 \pi)^{3}}\left[\frac{4}{3} Y_{j ; 0}(-p, t) V^{j}(p, t)-i p_{k} \alpha Y_{j}(-p, t) Z^{j k}(p, t)\right] \\
S_{A q} & =-\frac{1}{2} a T_{0}^{5} \int d t \frac{d^{3} p}{(2 \pi)^{3}}\left[\frac{1}{\alpha} Y_{j k ; 0}(-p, t) Z^{j k}(p, t)-2 i p^{k} Y_{j k}(-p, t) V^{j}(p, t)\right] \\
S_{I q} & =\frac{a T_{0}^{5}}{2 \alpha \tau} \int d t \frac{d^{3} p}{(2 \pi)^{3}} Y_{j k}(-p, t) Z^{j k}(p, t)
\end{aligned}
$$


and

$$
\begin{aligned}
S_{T c}= & -\sigma T_{0}^{4} \int d t \frac{d^{3} p}{(2 \pi)^{3}} \frac{d^{3} q}{(2 \pi)^{3}}\left[-\alpha Y_{j ; 0}(-p-q, t) Z_{k j}(q, t) V^{k}(p, t)\right. \\
& \left.-i(p+q)_{k} Y_{j}(-p-q, t)\left(\frac{4}{3} V^{j}(p, t) V^{k}(q, t)+Z^{j l}(p, t) Z_{l}^{k}(q, t)\right)\right] \\
S_{A c}= & -\frac{1}{2} a T_{0}^{5} \int d t \frac{d^{3} p}{(2 \pi)^{3}} \frac{d^{3} q}{(2 \pi)^{3}}\left[5 Y_{k j ; 0}(-p-q, t) V^{k}(p, t) V^{j}(q, t)\right. \\
& -i q_{l} \frac{2}{\alpha} Y_{k j}(-p-q, t) V^{k}(p, t) Z^{j l}(q, t) \\
& \left.-\frac{i}{\alpha}(p+q)_{l} Y_{j k}(-p-q, t)\left(Z^{j k}(q, t) V^{l}(p, t)+2 Z^{k l}(q, t) V^{j}(p, t)\right)\right]
\end{aligned}
$$

We may simplify these expressions by using the linear equations of motion derived from the quadratic terms, namely

$$
\begin{aligned}
0= & -\sigma T_{0}^{4} \frac{4}{3} Y_{j ; 0}(-p, t)+a T_{0}^{5} i p^{k} Y_{j k}(-p, t) \\
0= & -\frac{1}{2 \alpha} a T_{0}^{5} Y_{j k ; 0}(-p, t)+\frac{i}{2} \alpha \sigma T_{0}^{4}\left(p_{k} Y_{j}(-p, t)\right. \\
& \left.+p_{j} Y_{k}(-p, t)\right)+\frac{a T_{0}^{5}}{2 \alpha \tau} Y_{j k}(-p, t)
\end{aligned}
$$

where $O_{; 0}$ refers to the time derivative, to get

$$
\begin{aligned}
S_{T c}= & \int d t \frac{d^{3} p}{(2 \pi)^{3}} \frac{d^{3} q}{(2 \pi)^{3}}\left[\frac{3}{4} \alpha a T_{0}^{5} i p^{k} Y_{j k}(-p, t) Z_{l j}(q, t) V^{l}(p-q, t)\right. \\
& \left.+i \sigma T_{0}^{4} p_{k} Y_{j}(-p, t)\left(\frac{4}{3} V^{j}(p-q, t) V^{k}(q, t)+Z^{j l}(p-q, t) Z_{l}^{k}(q, t)\right)\right] \\
S_{A c}= & -\int d t \frac{d^{3} p}{(2 \pi)^{3}} \frac{d^{3} q}{(2 \pi)^{3}}\left[5\left[i \alpha \sigma T_{0}^{4} p_{k} Y_{j}(-p, t)+\frac{a T_{0}^{5}}{2 \alpha \tau} Y_{j k}(-p, t)\right] V^{k}(p-q, t) V^{j}(q, t)\right. \\
& -i q_{l} \frac{a}{\alpha} T_{0}^{5} Y_{k j}(-p, t) V^{k}(p-q, t) Z^{j l}(q, t) \\
& \left.-\frac{i}{\alpha} \frac{a}{2} T_{0}^{5} Y_{j k}(-p, t) p_{l}\left(Z^{j k}(q, t) V^{l}(p-q, t)+2 Z^{k l}(q, t) V^{j}(p-q, t)\right)\right]
\end{aligned}
$$

Finally, we discriminate between vector and proper tensor modes by writing

$$
\begin{aligned}
& Z^{j k}=i\left(p^{j} Z_{\mathrm{T}}^{k}+p^{k} Z_{\mathrm{T}}^{j}\right)+Z_{\mathrm{TT}}^{j k} \\
& Y^{j k}=i\left(p^{j} Y_{\mathrm{T}}^{k}+p^{k} Y_{\mathrm{T}}^{j}\right)+Y_{\mathrm{TT}}^{j k}
\end{aligned}
$$

where $p_{j} Z_{\mathrm{T}}^{j}=p_{j} Y_{\mathrm{T}}^{j}=p_{j} Z_{\mathrm{TT}}^{j k}=p_{j} Y_{\mathrm{TT}}^{j k}=0$. 


\subsection{The quadratic action}

After separating vector and tensor proper modes, the quadratic terms decouple. For the vectors we get

$$
\begin{aligned}
S_{N q V} & =i N \int d t \frac{d^{3} p}{(2 \pi)^{3}} p^{2} Y_{\mathrm{T} j}(-p, t) Y_{\mathrm{T}}^{j}(p, t) \\
S_{T q V} & =-\sigma T_{0}^{4} \int d t \frac{d^{3} p}{(2 \pi)^{3}}\left[\frac{4}{3} Y_{j ; 0}(-p, t) V^{j}(p, t)+p^{2} \alpha Y_{j}(-p, t) Z_{\mathrm{T}}^{j}(p, t)\right] \\
S_{A q V} & =-\frac{1}{2} a T_{0}^{5} \int d t \frac{d^{3} p}{(2 \pi)^{3}}\left[\frac{2}{\alpha} p^{2} Y_{\mathrm{T} j ; 0}(-p, t) Z_{\mathrm{T}}{ }^{j}(p, t)-2 p^{2} Y_{\mathrm{T} j}(-p, t) V^{j}(p, t)\right] \\
S_{I q V} & =\frac{a T_{0}^{5}}{\alpha \tau} \int d t \frac{d^{3} p}{(2 \pi)^{3}} p^{2} Y_{\mathrm{T} j}(-p, t) Z_{\mathrm{T}^{j}}(p, t)
\end{aligned}
$$

and for the tensors

$$
\begin{aligned}
& S_{N q T}=\frac{i N}{2} \int d t \frac{d^{3} p}{(2 \pi)^{3}} Y_{\mathrm{TT} j k}(-p, t) Y_{\mathrm{TT}}^{j k}(p, t) \\
& S_{A q T}=-\frac{1}{2} a T_{0}^{5} \int d t \frac{d^{3} p}{(2 \pi)^{3}}\left[\frac{1}{\alpha} Y_{\mathrm{TT} j k ; 0}(-p, t) Z_{\mathrm{TT}}{ }^{j k}(p, t)\right] \\
& S_{I q T}=\frac{a T_{0}^{5}}{2 \alpha \tau} \int d t \frac{d^{3} p}{(2 \pi)^{3}} Y_{\mathrm{TT} j k}(-p, t) Z_{\mathrm{TT}}{ }^{j k}(p, t)
\end{aligned}
$$

\subsection{The lowest order propagators}

We now return to the conformal fluid case, where the string of fields $\mathcal{X}^{K}$ may be split into physical vector fields $V^{j}$ and $Z_{\mathrm{T}}^{j}$, auxiliary vector fields $Y^{j}$ and $Y_{\mathrm{T}}^{j}$, the physical tensor proper field $Z_{\mathrm{TT}}{ }^{i j}$ and the auxiliary tensor proper field $Y_{\mathrm{TT}}{ }^{i j}$. The correlations between a physical and an auxiliary field yield the causal propagators; if the physical field is to the left, then it is a retarded propagator. The correlations between physical fields are the symmetric correlations in the theory; the correlations between auxiliary fields vanish identically. To lowest order, we obtain decoupled equations for correlations involving only vector fields and those involving only tensor fields.

\subsubsection{Causal vector correlations}

The causal vector correlations are $\left\langle V^{j} Y^{k}\right\rangle$ and $\left\langle Z_{\mathrm{T}}{ }^{j} Y^{k}\right\rangle$ on one hand, and $\left\langle V^{j} Y_{\mathrm{T}}{ }^{k}\right\rangle$ and $\left\langle Z_{\mathrm{T}}{ }^{j} Y_{\mathrm{T}}{ }^{k}\right\rangle$ on the other. These two pairs are decoupled from each other. They all have the structure

$$
\left\langle V^{j}(p, t) Y^{k}\left(q, t^{\prime}\right)\right\rangle=i(2 \pi)^{3} \delta(p+q) P^{j k}[p] G_{V Y}\left(p, t-t^{\prime}\right)
$$

where

$$
P^{j k}[p]=\delta^{j k}-\frac{p^{j} p^{k}}{p^{2}}
$$


The equations of motion for the $G_{V Y}, G_{Z_{\mathrm{T}} Y}$ pair are

$$
\begin{aligned}
\sigma T_{0}^{4}\left[\frac{4}{3} \frac{d}{d t} G_{V Y}-\alpha p^{2} G_{Z_{\mathrm{T}} Y}\right] & =\delta\left(t-t^{\prime}\right) \\
a T_{0}^{5} p^{2}\left[G_{V Y}+\frac{1}{\alpha} \frac{d}{d t} G_{Z_{\mathrm{T}} Y}\right]+\frac{a T_{0}^{5}}{\alpha \tau} p^{2} G_{Z_{\mathrm{T}} Y} & =0
\end{aligned}
$$

whose solution is

$$
\begin{aligned}
G_{V Y} & =\frac{e^{-\left(t-t^{\prime}\right) / 2 \tau}}{\sigma T_{0}^{4}}\left[\cos \omega\left(t-t^{\prime}\right)+\frac{\sin \omega\left(t-t^{\prime}\right)}{2 \omega \tau}\right] \Theta\left(t-t^{\prime}\right) \\
& =\frac{c_{V} p}{\sigma T_{0}^{4} \omega} e^{-\left(t-t^{\prime}\right) / 2 \tau} \cos \left[\omega\left(t-t^{\prime}\right)-\varphi_{p}\right] \Theta\left(t-t^{\prime}\right) \\
G_{Z_{\mathrm{T}} Y} & =\frac{-\alpha}{\omega} \frac{e^{-\left(t-t^{\prime}\right) / 2 \tau}}{\sigma T_{0}^{4}} \sin \omega\left(t-t^{\prime}\right) \Theta\left(t-t^{\prime}\right)
\end{aligned}
$$

where

$$
\begin{aligned}
\omega & =\sqrt{\frac{3}{4} \alpha^{2} p^{2}-\frac{1}{4 \tau^{2}}} \\
\varphi_{p} & =\tan ^{-1}\left(\frac{1}{2 \omega \tau}\right)=\frac{\pi}{2}-\tan ^{-1}(2 \omega \tau)
\end{aligned}
$$

$c_{V}=\sqrt{3} \alpha / 2$ is the propagation speed for vector modes (cfr. subsection 2.3), which in the limit we are interested satisfies $c_{V} \ll 1$.

For the second pair $\left\langle V^{j} Y_{\mathrm{T}}^{k}\right\rangle$ and $\left\langle Z_{\mathrm{T}}^{j} Y_{\mathrm{T}}^{k}\right\rangle$ we obtain

$$
\begin{gathered}
\sigma T_{0}^{4}\left[\frac{4}{3} \frac{d}{d t} G_{V Y_{\mathrm{T}}}-\alpha p^{2} G_{Z_{\mathrm{T}} Y_{\mathrm{T}}}\right]=0 \\
a T_{0}^{5} p^{2}\left[G_{V Y_{\mathrm{T}}}+\frac{1}{\alpha} \frac{d}{d t} G_{Z_{\mathrm{T}} Y_{\mathrm{T}}}\right]+\frac{a T_{0}^{5}}{\alpha \tau} p^{2} G_{Z_{\mathrm{T}} Y_{\mathrm{T}}}
\end{gathered}
$$

with solution

$$
\begin{aligned}
G_{V Y_{\mathrm{T}}} & =\frac{3 \alpha^{2}}{4 a T_{0}^{5} \omega} e^{-\left(t-t^{\prime}\right) / 2 \tau} \sin \omega\left(t-t^{\prime}\right) \Theta\left(t-t^{\prime}\right) \\
G_{Z_{\mathrm{T}} Y_{\mathrm{T}}} & =\frac{c_{V} \alpha}{a T_{0}^{5} p \omega} e^{-\left(t-t^{\prime}\right) / 2 \tau} \cos \left[\omega\left(t-t^{\prime}\right)+\varphi_{p}\right] \Theta\left(t-t^{\prime}\right)
\end{aligned}
$$

In summary, the causal correlations of vector fields all have the structure

$$
\left\langle X_{\alpha}^{j}(p, t) Y_{\beta}^{k}\left(q, t^{\prime}\right)\right\rangle=i(2 \pi)^{3} \delta(p+q) P^{j k}[p] C_{\alpha \beta} e^{-\left(t-t^{\prime}\right) / 2 \tau} \cos \left[\omega\left(t-t^{\prime}\right)-\varphi_{\alpha \beta}\right] \Theta\left(t-t^{\prime}\right)
$$

The different correlations are summarized in table 1.

\subsubsection{Causal tensor correlations}

The only causal tensor correlation is

$$
\left\langle Z_{\mathrm{TT}}^{i j}(p, t) Y_{\mathrm{TT} k l}\left(q, t^{\prime}\right)\right\rangle=i(2 \pi)^{3} \delta(p+q) S_{\mathrm{TT}}^{i j}{ }_{k l}[p] G_{Z_{\mathrm{TT}} Y_{\mathrm{TT}}}\left(p, t-t^{\prime}\right)
$$




\begin{tabular}{|c|c|c|c|}
\hline$X_{\alpha}$ & $Y_{\beta}$ & $C_{\alpha \beta}$ & $\varphi_{\alpha \beta}$ \\
\hline$V$ & $Y$ & $\left(c_{V} p\right) /\left(\sigma T_{0}^{4} \omega\right)$ & $\varphi_{p}$ \\
\hline$Z_{\mathrm{T}}$ & $Y$ & $(-\alpha) /\left(\sigma T_{0}^{4} \omega\right)$ & $\pi / 2$ \\
\hline$V$ & $Y_{\mathrm{T}}$ & $\left(3 \alpha^{2}\right) /\left(4 a T_{0}^{5} \omega\right)$ & $\pi / 2$ \\
\hline$Z_{\mathrm{T}}$ & $Y_{\mathrm{T}}$ & $\left(c_{V} \alpha\right)\left(a T_{0}^{5} p \omega\right)$ & $-\varphi_{p}$ \\
\hline
\end{tabular}

Table 1. Summary of vector causal propagators. $\omega$ and $\varphi_{p}$ are defined in eq. (4.45).

where

$$
S_{\mathrm{TT}}{ }^{i j}{ }_{k l}[p]=\frac{1}{2}\left[P_{k}^{i} P_{l}^{j}+P_{l}^{i} P_{k}^{j}-P^{i j} P_{k l}\right]
$$

It obeys the equation

$$
\frac{a T_{0}^{5}}{2 \alpha}\left[\frac{d}{d t}+\frac{1}{\tau}\right] G_{Z_{\mathrm{TT}} Y_{\mathrm{TT}}}=\delta\left(t-t^{\prime}\right)
$$

with solution

$$
G_{Z_{\mathrm{TT}} Y_{\mathrm{TT}}}=\frac{2 \alpha}{a T_{0}^{5}} e^{-\left(t-t^{\prime}\right) / \tau} \Theta\left(t-t^{\prime}\right)
$$

Observe that the tensor modes do not propagate, and their momentum dependence is trivial.

\subsubsection{Hadamard vector correlations}

The vector correlations are $\left\langle V^{j} V^{k}\right\rangle,\left\langle V^{j} Z_{\mathrm{T}}^{k}\right\rangle$ and $\left\langle Z_{\mathrm{T}}^{j} Z_{\mathrm{T}}^{k}\right\rangle$. They have the structure

$$
\left\langle V^{j}(p, t) V^{k}\left(q, t^{\prime}\right)\right\rangle=(2 \pi)^{3} \delta(p+q) P^{j k}[p] G_{1 V V}\left(p, t-t^{\prime}\right)
$$

They may be derived from eq. (3.39). For example

$$
G_{1 V V}\left(p, t-t^{\prime}\right)=2 N p^{2} \int^{\min \left(t, t^{\prime}\right)} d t^{\prime \prime} G_{V Y_{T}}\left(p, t-t^{\prime \prime}\right) G_{V Y_{T}}\left(p, t^{\prime}-t^{\prime \prime}\right)
$$

Explicitly,

$$
\begin{aligned}
G_{1 V V}\left(p, t-t^{\prime}\right) & =\frac{3}{4 \sigma T_{0}^{3}} e^{-\left|t-t^{\prime}\right| / 2 \tau}\left[\cos \omega\left|t-t^{\prime}\right|+\frac{\sin \omega\left|t-t^{\prime}\right|}{2 \omega \tau}\right] \\
& =\frac{3 c_{V} p}{4 \sigma T_{0}^{3} \omega} e^{-\left|t-t^{\prime}\right| / 2 \tau} \cos \left[\omega\left|t-t^{\prime}\right|-\varphi_{p}\right]
\end{aligned}
$$

Similarly

$$
G_{1 V Z_{\mathrm{T}}}\left(p, t-t^{\prime}\right)=2 N p^{2} \int^{\min \left(t, t^{\prime}\right)} d t^{\prime \prime} G_{V Y_{\mathrm{T}}}\left(p, t-t^{\prime \prime}\right) G_{Z_{\mathrm{T}} Y_{\mathrm{T}}}\left(p, t^{\prime}-t^{\prime \prime}\right)
$$

Now, from the equations for the propagators

$$
G_{1 V Z_{\mathrm{T}}}\left(p, t-t^{\prime}\right)=2 N p^{2} \int^{\min \left(t, t^{\prime}\right)} d t^{\prime \prime} G_{V Y_{\mathrm{T}}}\left(p, t-t^{\prime \prime}\right) \frac{4}{3 \alpha p^{2}} \frac{d}{d t^{\prime}} G_{V Y_{\mathrm{T}}}\left(p, t^{\prime}-t^{\prime \prime}\right)
$$




\begin{tabular}{|c|c|c|c|c|}
\hline$X_{\alpha}$ & $X_{\beta}$ & $C_{1 \alpha \beta}$ & $\varphi_{1 \alpha \beta}$ & $q_{\alpha \beta}$ \\
\hline$V$ & $V$ & $\left(3 c_{V} p\right) /\left(4 \sigma T_{0}^{3} \omega\right)$ & $\varphi_{p}$ & 0 \\
\hline$V$ & $Z_{\mathrm{T}}$ & $(3 \alpha) /\left(4 \sigma T_{0}^{3} \omega\right)$ & $\pi / 2$ & 1 \\
\hline$Z_{\mathrm{T}}$ & $Z_{\mathrm{T}}$ & $\left(c_{V}\right) /\left(\sigma T_{0}^{3} \omega p\right)$ & $-\varphi_{p}$ & 0 \\
\hline
\end{tabular}

Table 2. Summary of vector correlations. $\varphi_{p}$ is defined in eq. (4.45).

Since the integrand vanishes at the upper limit, we find

$$
\begin{aligned}
G_{1 V Z_{\mathrm{T}}}\left(p, t-t^{\prime}\right) & =\frac{4}{3 \alpha p^{2}} \frac{d}{d t^{\prime}} G_{1 V V}\left(p, t-t^{\prime}\right) \\
& =\frac{3 \alpha}{4 \sigma T_{0}^{3} \omega} e^{-\left|t-t^{\prime}\right| / 2 \tau} \sin \omega\left|t-t^{\prime}\right| \operatorname{sign}\left(t-t^{\prime}\right)
\end{aligned}
$$

The correlation $G_{1 Z_{\mathrm{T}} Z_{\mathrm{T}}}$ is even in $t-t^{\prime}$, and so there is no loss of generality in assuming $t>t^{\prime}$. In this case, we get

$$
\begin{aligned}
G_{1 Z_{\mathrm{T}} Z_{\mathrm{T}}}\left(p, t-t^{\prime}\right) & =\frac{4}{3 \alpha p^{2}} \frac{d}{d t} G_{1 V Z_{\mathrm{T}}}\left(p, t-t^{\prime}\right) \\
& =\frac{1}{\sigma T_{0}^{3} p^{2}} e^{-\left|t-t^{\prime}\right| / 2 \tau}\left[\cos \omega\left|t-t^{\prime}\right|-\frac{\sin \omega\left|t-t^{\prime}\right|}{2 \omega \tau}\right] \\
& =\frac{c_{V}}{\sigma T_{0}^{3} \omega p} e^{-\left|t-t^{\prime}\right| / 2 \tau} \cos \left[\omega\left|t-t^{\prime}\right|+\varphi_{p}\right]
\end{aligned}
$$

In summary, the structure of vector correlations is

$$
\left\langle X_{\alpha}^{j}(p, t) X_{\beta}^{k}\left(q, t^{\prime}\right)\right\rangle=(2 \pi)^{3} \delta(p+q) P^{j k}[p] C_{1 \alpha \beta} e^{-\left|t-t^{\prime}\right| / 2 \tau} \cos \left[\omega\left|t-t^{\prime}\right|-\varphi_{1 \alpha \beta}\right]\left(\operatorname{sign}\left(t-t^{\prime}\right)\right)^{q_{\alpha \beta}}
$$

They are summarized in table 2 .

\subsubsection{Hadamard tensor correlations}

The remaining correlation is trivial. It has the structure

$$
\left\langle Z_{\mathrm{TT}}^{j k}(p, t) Z_{\mathrm{TT}}^{l m}\left(q, t^{\prime}\right)\right\rangle=(2 \pi)^{3} \delta(p+q) S_{\mathrm{TT}^{j k l m}}[p] G_{1 Z_{\mathrm{TT}} Z_{\mathrm{TT}}}\left(p, t-t^{\prime}\right)
$$

where the projector $S_{\mathrm{TT}}$ is defined in eq. (4.51), and

$$
\begin{aligned}
G_{1 Z_{\mathrm{TT}} Z_{\mathrm{TT}}} & =N\left(\frac{2 \alpha}{a T_{0}^{5}}\right)^{2} \frac{\tau}{2} e^{-\left|t-t^{\prime}\right| / \tau} \\
& =\frac{2}{\sigma T_{0}^{3}} e^{-\left|t-t^{\prime}\right| / \tau}
\end{aligned}
$$

We may check that in the limit $\tau \rightarrow 0$ we recover Landau-Lifshitz theory. Indeed in this limit we may approximate

$$
G_{1 Z_{\mathrm{TT}} Z_{\mathrm{TT}}}=\frac{4 \tau}{\sigma T_{0}^{3}} \delta\left(t-t^{\prime}\right)
$$


In this limit, the self correlation for the tensor proper part of the viscous EMT (cfr. eq. (2.54)) is

$$
\begin{aligned}
\left\langle\Pi_{\mathrm{TT}^{j k}}^{j k}(p, t) \Pi_{\mathrm{TT}}^{l m}\left(q, t^{\prime}\right)\right\rangle & =\alpha^{2} \sigma^{2} T_{0}^{8}\left\langle Z_{\mathrm{TT}^{j k}}(p, t) Z_{\mathrm{TT}}^{l m}\left(q, t^{\prime}\right)\right\rangle \\
& =(2 \pi)^{3} \delta(p+q) S_{\mathrm{TT}^{j k l m}}[p] 4 T_{0} \eta \delta\left(t-t^{\prime}\right)
\end{aligned}
$$

where we have used eq. (2.62). We thus recover the Landau-Lifshitz result [51, 52] in this limit.

\section{Nonlinear fluctuations around equilibrium}

In this work we shall show the application of the field theory technics to a consistent causal theory. We choose to focus on the interaction between tensor and vector modes. In this section we compute the one-loop corrections to the tensor propagators found above, and apply the results to derive the corresponding fluctuations in the energy and entropy densities. To this purpose we need the cubic terms in the "classical" action eq. (4.1).

Considering heavy-ion collisions, we may ask at which stage of the fireball evolution are the loop corrections significative. We shall show below (eq. (5.45)) that this holds for $p>p_{T} \sim T_{0}\left(T_{0} \tau\right)^{-3 / 8}$. Since we work within the free-streaming approximation, it implies that the spatial correlation at equal times is non-trivial for distances $r<p_{T}^{-1}<c_{V} \tau<\tau$. These are the relevant scales at the very early stages of a heavy-ion collision.

\subsection{Cubic terms in the "classical" action}

We shall not need the cubic terms which do not contain tensor modes. For those which contain tensor fields, we distinguish

(a) Terms that only contain $Y_{\mathrm{TT} j k}(-p, t)$ : these terms naturally split into two

$$
\begin{aligned}
S_{Y_{\mathrm{TT}}} & =S_{Y_{\mathrm{TT}} V V}+S_{Y_{\mathrm{TT}} V Z_{\mathrm{T}}} \\
S_{Y_{\mathrm{TT}} V V} & =-\frac{5 a T_{0}^{5}}{2 \alpha \tau} \int d t \frac{d^{3} p}{(2 \pi)^{3}} \frac{d^{3} q}{(2 \pi)^{3}} D_{j k r s}(p, q) Y_{\mathrm{TT}}{ }^{j k}(-p, t) V^{r}(p-q, t) V^{s}(q, t) \\
S_{Y_{\mathrm{TT}} V Z_{\mathrm{T}}} & =-\frac{a T_{0}^{5}}{\alpha} \int d t \frac{d^{3} p}{(2 \pi)^{3}} \frac{d^{3} q}{(2 \pi)^{3}} E_{j k r s}(p, q) Y_{\mathrm{TT}}^{j k}(-p, t) V^{r}(p-q, t) Z_{\mathrm{T}}{ }^{s}(q, t)
\end{aligned}
$$

where

$$
\begin{aligned}
& D_{j k r s}(p, q)=\delta_{r k} \delta_{j s} \\
& E_{j k r s}(p, q)=\left(q^{2}+p_{l} q^{l}\right) \delta_{s j} \delta_{k r}+q^{k}\left(\delta_{j s} p_{r}+p_{s} \delta_{j r}\right)
\end{aligned}
$$

(b) Terms that only contain $Z_{\mathrm{TT} l m}(q, t)$ : these terms also split as

$$
\begin{aligned}
S_{Z_{\mathrm{TT}}} & =S_{Y_{\mathrm{T}} V Z_{\mathrm{TT}}}+S_{Y Z_{\mathrm{T}} Z_{\mathrm{TT}}} \\
S_{Y_{\mathrm{T}} V Z_{\mathrm{TT}}} & =\frac{a T_{0}^{5}}{\alpha} \int d t \frac{d^{3} p}{(2 \pi)^{3}} \frac{d^{3} q}{(2 \pi)^{3}} F_{r s l m}(p, q) Y_{\mathrm{T}}^{r}(-p, t) V^{s}(p-q, t) Z_{\mathrm{TT}}^{l m}(q, t) \\
S_{Y Z_{\mathrm{T}} Z_{\mathrm{TT}}} & =-\sigma T_{0}^{4} \int d t \frac{d^{3} p}{(2 \pi)^{3}} \frac{d^{3} q}{(2 \pi)^{3}} G_{r s l m}(p, q) Y^{r}(-p, t) Z_{\mathrm{T}}^{s}(p-q, t) Z_{\mathrm{TT}}^{l m}(q, t)
\end{aligned}
$$


where

$$
\begin{aligned}
& F_{r s l m}(p, q)=\frac{3}{4} \alpha^{2} p^{2} \delta_{m r} \delta_{l s}+2 p^{l} \delta_{m r} p_{s}+p^{m} p_{l} \delta_{r s} \\
& G_{r s l m}(p, q)=p_{m}\left((p-q)^{r} \delta_{l s}+(p-q)^{l} \delta_{r s}\right)+\delta_{r m}\left(p_{s}(p-q)^{l}+p_{k}(p-q)^{k} \delta_{s l}\right)
\end{aligned}
$$

We shall not need the explicit form of the remaining terms:

(c) terms that contain both $Y_{\mathrm{TT} j k}(-p, t)$ and $Z_{\mathrm{TT} l m}(q, t)$,

(d) terms quadratic in $Z_{\mathrm{TT} l m}(q, t)$.

\subsection{Tensor self-energy}

We now turn to the derivation of the self energy for tensor modes

$$
\Sigma_{j k l m}\left(-p, q, t-t^{\prime}\right)=2 \frac{\delta \Gamma_{Q}}{\delta\left\langle Y_{\mathrm{TT}^{j k}}(-p, t) Z_{\mathrm{TT}}^{l m}\left(q, t^{\prime}\right)\right\rangle}
$$

We may split the self energy into two contributions, one with only vector propagators in internal lines, and the other with one vector and one tensor modes. Our goal is to derive the momentum dependence of the self-energy and the noise kernel. Now, because the lowest order tensor propagators are momentum independent, the Feynman graphs containing them are momentum independent too. For this reason we shall not compute them.

The self-energy, considering only the Feynman graphs with vector propagators in internal lines, is

$$
\Sigma_{j k l m}^{(V)}\left(-p, q, t-t^{\prime}\right)=i\left\langle\frac{\delta\left(S_{Y_{\mathrm{TT}} V V}+S_{Y_{\mathrm{TT}} V Z_{\mathrm{T}}}\right)}{\delta Y_{\mathrm{TT}}^{j k}(-p, t)} \frac{\delta\left(S_{Y_{\mathrm{T}} V Z_{\mathrm{TT}}}+S_{Y Z_{\mathrm{T}} Z_{\mathrm{TT}}}\right)}{\delta Z_{\mathrm{TT}}^{l m}\left(q, t^{\prime}\right)}\right\rangle
$$

It is understood that after computing the functional derivatives one must project back onto tensor proper modes. The terms involving $S_{Y_{\mathrm{TT}} V V}$ are suppressed by one power of $\tau$ and will not be computed. Let us begin with

$$
\begin{aligned}
\Sigma_{j k l m}^{(V 1)}\left(-p, q, t-t^{\prime}\right)= & i\left\langle\frac{\delta S_{Y_{\mathrm{TT}} V Z_{\mathrm{T}}}}{\delta Y_{\mathrm{TT}}^{j k}(-p, t)} \frac{\delta S_{Y_{\mathrm{T}} V Z_{\mathrm{TT}}}}{\delta Z_{\mathrm{TT}}^{l m}\left(q, t^{\prime}\right)}\right\rangle \\
= & -i \frac{a^{2} T_{0}^{10}}{\alpha^{2}} \int \frac{d^{3} q^{\prime}}{(2 \pi)^{6}} \frac{d^{3} p^{\prime}}{(2 \pi)^{6}} E_{j k r s}\left(p, q^{\prime}\right) F_{u v l m}\left(p^{\prime}, q\right) \\
& \left\{\left\langle V^{r}\left(p-q^{\prime}, t\right) Y_{\mathrm{T}}^{u}\left(-p^{\prime}, t^{\prime}\right)\right\rangle\left\langle Z_{\mathrm{T}}{ }^{s}\left(q^{\prime}, t\right) V^{v}\left(p^{\prime}-q, t^{\prime}\right)\right\rangle\right. \\
& \left.+\left\langle V^{r}\left(p-q^{\prime}, t\right) V^{v}\left(p^{\prime}-q, t^{\prime}\right)\right\rangle\left\langle Z_{\mathrm{T}}{ }^{s}\left(q^{\prime}, t\right) Y_{\mathrm{T}}^{u}\left(-p^{\prime}, t^{\prime}\right)\right\rangle\right\}
\end{aligned}
$$

Observe that $\Sigma_{j k l m}^{(V 1)}$ vanishes unless $t \geq t^{\prime}$, which we assume. Moreover, the graph in the second line of the right hand side vanishes when $t=t^{\prime}$ and we shall not compute it. We then have

$$
\Sigma_{j k l m}^{(V 1)}\left(-p, q, t-t^{\prime}\right)=\frac{9 \alpha a T_{0}^{2}}{16 \sigma} \delta(p-q)(2 \pi)^{-6} e^{-\left(t-t^{\prime}\right) / \tau} \Theta\left(t-t^{\prime}\right) \sigma_{j k l m}^{(V 1)}
$$


where

$$
\begin{aligned}
\sigma_{j k l m}^{(V 1)}= & \int d^{3} q^{\prime} E_{j k r s}\left(p, q^{\prime}\right) F_{u v l m}\left(q^{\prime}, p\right) P^{r v}\left[p-q^{\prime}\right] P^{s u}\left[q^{\prime}\right] \\
& \frac{\left|p-q^{\prime}\right|}{q^{\prime} \omega_{q^{\prime}} \omega_{p-q^{\prime}}} \cos \left[\omega_{p-q^{\prime}}\left(t-t^{\prime}\right)-\varphi_{p-q^{\prime}}\right] \cos \left[\omega_{q^{\prime}}\left(t-t^{\prime}\right)+\varphi_{q^{\prime}}\right]
\end{aligned}
$$

On dimensional grounds, we see that the tensor self energy has units of $T^{4}$ as it should. $\sigma_{j k l m}^{(V 1)}$ has units of $p^{5}$.

To obtain the true self energy we must project back on the transverse components, symmetric and traceless in $j k$ and $l m$. If $p$ lies in the $z$ direction, this means we only need $\sigma_{a b c d}^{(V 1)}$, where the indices run from 1 to 2 . Since there are no preferred directions, we will obtain

$$
\sigma_{a b c d}^{(V 1)}=A \delta_{a c} \delta_{b d}+B \delta_{a d} \delta_{b c}+C \delta_{a b} \delta_{c d}
$$

Symmetrization on $a b$ yields

$$
\sigma_{a b c d}^{(V 1)} \rightarrow \frac{1}{2}(A+B)\left(\delta_{a c} \delta_{b d}+\delta_{a d} \delta_{b c}\right)+C \delta_{a b} \delta_{c d}
$$

and removing the trace on $a b$ we get

$$
\sigma_{a b c d}^{(V 1)} \rightarrow(A+B) S_{\mathrm{TT} a b c d} \frac{1}{2}\left(\delta_{a c} \delta_{b d}+\delta_{a d} \delta_{b c}-\delta_{a b} \delta_{c d}\right)
$$

where $S_{\mathrm{TT} a b c d}$ is the restriction to the case where $p$ is on the third direction of the projector eq. (4.51). This means that the physical self energy takes the form

$$
\Sigma_{i j l m}^{(V 1)}\left(-p, q, t-t^{\prime}\right)=\frac{9 \alpha a T_{0}^{2}}{16 \sigma} \delta(p-q)(2 \pi)^{-6} e^{-\left|t-t^{\prime}\right| / \tau} \Theta\left(t-t^{\prime}\right) \sigma_{\mathrm{phys}}^{(V 1)} S_{\mathrm{TT} i j l m}
$$

where

$$
\sigma_{\text {phys }}^{(V 1)}=\frac{1}{2} \int d^{3} q^{\prime} W\left[p, q^{\prime}\right] \frac{\left|p-q^{\prime}\right|}{\omega_{p-q^{\prime}}} \cos \left[\omega_{p-q^{\prime}}\left(t-t^{\prime}\right)-\varphi_{p-q^{\prime}}\right] \cos \left[\omega_{q^{\prime}}\left(t-t^{\prime}\right)+\varphi_{q^{\prime}}\right]
$$

with

$$
W\left[p, q^{\prime}\right]=\frac{1}{q^{\prime} \omega_{q^{\prime}}} S_{\mathrm{TT}}^{i j k l}[p] E_{j k r s}\left(p, q^{\prime}\right) F_{u v l m}\left(q^{\prime}, p\right) P^{r v}\left[p-q^{\prime}\right] P^{s u}\left[q^{\prime}\right]
$$

observe that $W$ has units of $p^{2}$.

To proceed, we shall make an important simplification. As we have seen, the "classical" equations for the correlations eqs. (4.42), (4.46), (4.52) are local in time. We assume that the main loop corrections to these equations are those that are local in time too. Therefore we shall seek only the singular terms in the self energy and the noise kernel, namely the terms which are proportional to $\delta\left(t-t^{\prime}\right)$. Since the propagators themselves are regular functions, any such singular term can only result from the asymptotic large $\left|q^{\prime}\right|$ region of the integration domain. In this region $\omega_{q^{\prime}}$ and $\omega_{p-q^{\prime}}$ are both real. In principle different schemes, such as BRSSS [3, 114], DNMR [115], Anisotropic Hydrodynamics [4] or DTTs, may lead to different UV behaviors and, moreover, different procedures can be adopted 
to regularize the large momentum contribution to the Feynman graphs. Therefore, the ensuing discussion is predicated on our choice of DTTs and dimensional regularization.

To compute $\sigma_{\text {phys }}^{(V 1)}$ we keep only the terms which are slow and not zero in the coincidence limit

$$
\begin{aligned}
& \cos \left[\omega_{p-q^{\prime}}\left(t-t^{\prime}\right)-\varphi_{p-q^{\prime}}\right] \cos \left[\omega_{q^{\prime}}\left(t-t^{\prime}\right)+\varphi_{q^{\prime}}\right] \\
& \simeq \frac{1}{2} \cos \left[\left(\omega_{p-q^{\prime}}-\omega_{q^{\prime}}\right)\left(t-t^{\prime}\right)\right] \cos \left[\varphi_{p-q^{\prime}}\right] \cos \left[\varphi_{q^{\prime}}\right]
\end{aligned}
$$

So

$$
\sigma_{\text {phys }}^{(V 1)} \approx \frac{1}{4} \int d^{3} q^{\prime} W\left[p, q^{\prime}\right] \frac{\left|p-q^{\prime}\right|}{\omega_{p-q^{\prime}}} \cos \left[\left(\omega_{p-q^{\prime}}-\omega_{q^{\prime}}\right)\left(t-t^{\prime}\right)\right] \cos \left[\varphi_{p-q^{\prime}}\right] \cos \left[\varphi_{q^{\prime}}\right]
$$

Assume again the $p^{j}=(0,0, p)$. Then $\omega_{p-q^{\prime}}-\omega_{q^{\prime}}$ vanishes when $q^{\prime}=\left(q_{T}, p / 2\right)$, with $q_{T}$ in the $x, y$ plane. We write $q^{\prime}=\left(q_{T}, p / 2+\delta q_{3}\right)$ to get (where explicit, $\left.q^{\prime}=\left(q_{T}, p / 2\right)\right)$

$$
\omega_{p-q^{\prime}}-\omega_{q^{\prime}} \approx-\frac{c_{V}^{2} p}{\omega_{q^{\prime}}} \delta q_{3}
$$

Evaluating the prefactors at $\delta q_{3}=0$ we may integrate over $\delta q_{3}$ to obtain the singular part of the self-energy

$$
\sigma_{\text {phys }}^{(V 1)} \approx \frac{\pi}{2 c_{V}^{2}} \delta\left(t-t^{\prime}\right) \int d^{2} q_{T} W\left[p, q^{\prime}\right] \frac{q^{\prime}}{p} \cos ^{2}\left[\varphi_{q^{\prime}}\right]
$$

where, in the free-streaming limit $\tau \rightarrow \infty$,

$$
\cos ^{2}\left[\varphi_{q^{\prime}}\right]=\frac{1+\cos \left[2 \varphi_{q^{\prime}}\right]}{2}=1-\frac{1}{4 \omega_{q^{\prime}}^{2} \tau^{2}} \approx 1
$$

The remaining task is to compute (we neglect terms proportional to $\alpha^{2}$ )

$$
\begin{aligned}
J^{(V 1)} & =\frac{\pi}{2 c_{V}^{2}} \int d^{2} q_{T} W\left[p, q^{\prime}\right] \frac{q^{\prime}}{p} \\
& =\frac{\pi}{2 p c_{V}^{3}} \int \frac{d^{2} q_{T}\left[-q_{T}^{8}+\frac{5}{8} q_{T}^{6} p^{2}-\frac{1}{8} q_{T}^{4} p^{4}+\frac{17}{128} q_{T}^{2} p^{6}\right]}{\left(q_{T}^{2}+\frac{p^{2}}{4}\right)^{2}\left(q_{T}^{2}+\frac{p^{2}}{4}-\frac{1}{4 c_{V}^{2} \tau^{2}}\right)^{1 / 2}}
\end{aligned}
$$

We have integrals of the form

$$
\begin{aligned}
s^{(n)}(p) & =\int \frac{d^{2} q_{T}\left(q_{T}^{2}\right)^{n}}{\left(q_{T}^{2}+\frac{p^{2}}{4}\right)^{2}\left(q_{T}^{2}+\frac{p^{2}}{4}-\frac{1}{4 c_{V}^{2} \tau^{2}}\right)^{1 / 2}} \\
& =\frac{\Gamma[5 / 2]}{\Gamma[2] \Gamma[1 / 2]} \int_{0}^{1} d x(1-x) x^{-1 / 2} \int \frac{d^{2} q_{T} q_{T}^{2 n}}{\left(q_{T}^{2}+\frac{p^{2}}{4}-\frac{x}{4 c_{V}^{2} \tau^{2}}\right)^{5 / 2}}
\end{aligned}
$$


We compute them in the scheme of dimensional regularization in $D=2-\epsilon$ dimensions. Following $[116,117]$ we get

$$
\begin{aligned}
s^{n}(p)= & \pi^{1-\epsilon / 2} \frac{\Gamma[1-\epsilon / 2+n] \Gamma[3 / 2+\epsilon / 2-n]}{\Gamma[2] \Gamma[1 / 2] \Gamma[1-\epsilon / 2]} \\
& \mu^{\epsilon} \int_{0}^{1} d x(1-x) x^{-1 / 2}\left[\frac{1}{4}\left(p^{2}-\frac{x}{c_{V}^{2} \tau^{2}}\right)\right]^{n-\epsilon / 2-3 / 2} .
\end{aligned}
$$

As we see the limit $\epsilon \rightarrow 0$ is well defined for any integer $n$, so we take $\epsilon=0(D=2)$ straightforwardly. Further in the free-streaming limit $\tau \rightarrow \infty$, we get

$$
\begin{aligned}
J^{(V 1)} & =\frac{493 \pi^{2}}{480} \frac{p^{4}}{c_{V}^{3}} \\
\Sigma_{i j l m}^{(V 1)}\left(-p, q, t-t^{\prime}\right) & =\frac{493 \sqrt{3} \pi^{2}}{2560} \frac{1}{(2 \pi)^{6}} \frac{a T_{0}^{2} p^{4}}{c_{V}^{2} \sigma} \delta(p-q) \delta\left(t-t^{\prime}\right) S_{\mathrm{TT} i j l m}(p)
\end{aligned}
$$

We now consider the other graph

$$
\Sigma_{j k l m}^{(V 2)}\left(-p, q, t-t^{\prime}\right)=i\left\langle\frac{\delta S_{Y_{\mathrm{TT}} V Z_{\mathrm{T}}}}{\delta Y_{\mathrm{TT}}^{j k}(-p, t)} \frac{\delta S_{Y Z_{\mathrm{T}} Z_{\mathrm{TT}}}}{\delta Z_{\mathrm{TT}}^{l m}\left(q, t^{\prime}\right)}\right\rangle
$$

Repeating the same steps as in the previous case we get

$$
\Sigma_{j k l m}^{(V 2)}\left(-p, q, t-t^{\prime}\right)=-\frac{a T_{0}^{2} c_{V}^{2} e^{-\left(t-t^{\prime}\right) / \tau}}{(2 \pi)^{6} \alpha \sigma} \Theta\left(t-t^{\prime}\right) \delta(p-q) \sigma_{\mathrm{phys}}^{(V 2)}\left(p, t-t^{\prime}\right) S_{\mathrm{TT} j k l m}(p) .
$$
limit

To compute $\sigma_{\text {phys }}^{(V 2)}$ we keep only the terms which are slow and not zero in the coincidence

$$
\sigma_{\text {phys }}^{(V 2)}\left(p, t-t^{\prime}\right) \simeq \frac{\pi}{2 c_{V}^{2}} \delta\left(t-t^{\prime}\right) \int \frac{d^{2} k_{\perp}}{p \omega_{k}} \frac{\left[\frac{1}{2} k_{\perp}^{8}+k_{\perp}^{6} p^{2}+\frac{9}{32} k_{\perp}^{4} p^{4}+\frac{9}{64} k_{\perp}^{2} p^{6}-\frac{3}{128} p^{8}\right]}{\left(k_{\perp}^{2}+\frac{p^{2}}{4}\right)^{2}}
$$

Finally, in the free-streaming limit, we get

$$
\sigma_{\text {phys }}^{(V 2)}\left(p, t-t^{\prime}\right)=\frac{17 \pi^{2}}{60} \frac{p^{4}}{c_{V}^{3}} \delta\left(t-t^{\prime}\right)
$$

and

$$
\Sigma_{j k l m}^{(V 2)}\left(-p, q, t-t^{\prime}\right)=-\frac{17 \sqrt{3}}{240} \frac{\pi^{2}}{(2 \pi)^{6}} \frac{a T_{0}^{2} p^{4}}{c_{V}^{2} \sigma} \delta(p-q) \delta\left(t-t^{\prime}\right) S_{\mathrm{TT} j k l m}(p)
$$

The total physical self-energy induced by vector fluctuations reads, in the freestreaming limit,

$$
\Sigma_{j k l m}^{(V)}\left(-p, q, t-t^{\prime}\right)=\frac{\gamma_{\Sigma}}{(2 \pi)^{3}} \frac{\sqrt{3} a T_{0}^{2} p^{4}}{4 c_{V}^{2} \sigma} \delta(p-q) \delta\left(t-t^{\prime}\right) S_{\mathrm{TT} j k l m}(p)
$$

where

$$
\gamma_{\Sigma}=\frac{187}{384} \frac{\pi^{2}}{(2 \pi)^{3}}
$$




\subsection{Tensor noise-kernel}

The derivation of the noise kernel begins with

$$
N_{j k l m}\left(-p, q, t-t^{\prime}\right)=-2 i \frac{\delta \Gamma_{Q}}{\delta\left\langle Y_{\mathrm{TT}}^{j k}(-p, t) Y_{\mathrm{TT}}^{l m}\left(q, t^{\prime}\right)\right\rangle}
$$

As before, we may split the noise kernel into two contributions, one with only vector propagators in internal lines, and the other with one vector and one tensor propagator. Since the latter is momentum independent, we shall compute the former only:

$$
N_{j k l m}^{(V)}\left(-p, q, t-t^{\prime}\right)=\left\langle\frac{\delta\left(S_{Y_{\mathrm{TT}} V V}+S_{Y_{\mathrm{TT}} V Z_{\mathrm{T}}}\right)}{\delta Y_{\mathrm{TT}}{ }^{j k}(-p, t)} \frac{\delta\left(S_{Y_{\mathrm{TT}} V V}+S_{Y_{\mathrm{TT}} V Z_{\mathrm{T}}}\right)}{\delta Y_{\mathrm{TT}}{ }^{l m}\left(q, t^{\prime}\right)}\right\rangle
$$

It is understood that after computing the functional derivatives one must project back onto tensor proper modes.

The terms involving $S_{Y_{\mathrm{TT}} V V}$ are suppressed by one power of $\tau$ and will not be computed. In consequence the noise kernel reads

$$
N_{j k l m}^{(V)}\left(-p, q, t-t^{\prime}\right)=\left\langle\frac{\delta S_{Y_{\mathrm{TT}} V Z_{\mathrm{T}}}}{\delta Y_{\mathrm{TT}}^{j k}(-p, t)} \frac{\delta S_{Y_{\mathrm{TT}} V Z_{\mathrm{T}}}}{\delta Y_{\mathrm{TT}}^{l m}\left(q, t^{\prime}\right)}\right\rangle
$$

As before, we seek the singular part of the noise kernel. The calculation follows the same steps as the self-energy computation. We get

$$
N_{j k l m}^{(V)}\left(-p, q, t-t^{\prime}\right)=\left(\frac{a T_{0}^{5}}{\alpha}\right)^{2} \frac{3 c_{V}^{2} e^{-\left|t-t^{\prime}\right| / \tau}}{4(2 \pi)^{6} T_{0}^{6} \sigma^{2}} \delta(p-q) S_{\mathrm{TT} j k l m}(p) n_{\mathrm{phys}}^{(V)}
$$

To compute $n_{\text {phys }}^{(V)}$ we keep only the terms which are slow and not zero in the coincidence limit

$$
n_{\mathrm{phys}}^{(V)} \simeq \frac{\pi}{2 c_{V}^{2}} \delta\left(t-t^{\prime}\right) \int \frac{d^{2} k_{\perp}}{p \omega_{k}} \frac{\left[\frac{1}{2} k_{\perp}^{8}+\frac{9}{4} k_{\perp}^{6} p^{2}+\frac{45}{32} k_{\perp}^{4} p^{4}+\frac{15}{32} k_{\perp}^{2} p^{6}+\frac{9}{128} p^{8}\right]}{\left(k_{\perp}^{2}+\frac{p^{2}}{4}\right)^{2}}
$$

Finally, in the free-streaming limit, we get

$$
n_{\mathrm{phys}}^{(V)}\left(p, t-t^{\prime}\right)=\frac{73 \pi^{2}}{240} \frac{p^{4}}{c_{V}^{3}} \delta\left(t-t^{\prime}\right)
$$

and

$$
N_{j k l m}^{(V)}\left(-p, q, t-t^{\prime}\right)=\frac{\gamma_{N}}{(2 \pi)^{3}} \frac{3 a^{2} T_{0}^{4} p^{4}}{4 c_{V}^{3} \sigma^{2}} \delta(p-q) \delta\left(t-t^{\prime}\right) S_{\mathrm{TT} j k l m}(p)
$$

where

$$
\gamma_{N}=\frac{73 \pi^{2}}{320(2 \pi)^{3}}
$$




\subsection{Nonlinear tensor correlations}

Adding the singular self-energy term to the classical equation (4.52) we get the one-loop corrected equation for the causal tensor propagator

$$
\frac{a T_{0}^{5}}{2 \alpha}\left[\frac{d}{d t}+\frac{1}{\tau_{1}}\right] G_{Z_{\mathrm{TT}} Y_{\mathrm{TT}}}=\delta\left(t-t^{\prime}\right)
$$

where

$$
\frac{1}{\tau_{1}}=\frac{1}{\tau}\left[1+\gamma_{\Sigma}\left(\frac{p}{p_{\mathrm{T}}}\right)^{4}\right]
$$

and

$$
p_{\mathrm{T}}=\left(\frac{c_{V} \sigma T_{0}^{3}}{\tau}\right)^{1 / 4}=\left(\frac{p_{\mathrm{L}}^{3}}{c_{V} \tau}\right)^{1 / 4}
$$

with $p_{\mathrm{L}}$ in (3.27), being the largest value of $p$ that makes the loop expansion consistent. Since $c_{V} \tau \sim \sqrt{\tau / T}$, in the free-streaming limit $(\tau \rightarrow \infty)$ we get $p_{\mathrm{T}} \ll p_{\mathrm{L}}$.

Therefore the causal correlation becomes (cfr. eq. (4.53))

$$
G_{Z_{\mathrm{TT}} Y_{\mathrm{TT}}}=\frac{2 \alpha}{a T_{0}^{5}} e^{-\left(t-t^{\prime}\right) / \tau_{1}} \Theta\left(t-t^{\prime}\right),
$$

and the symmetric tensor correlation, analogous to the eq. (4.63), reads

$$
\begin{aligned}
G_{1 Z_{\mathrm{TT}} Z_{\mathrm{TT}}} & =\left[N+\gamma_{N} \frac{3 a^{2} T_{0}^{4} p^{4}}{4 c_{V}^{3} \sigma^{2}}\right]\left(\frac{2 \alpha}{a T_{0}^{5}}\right)^{2} \frac{\tau_{1}}{2} e^{-\left|t-t^{\prime}\right| / \tau_{1}} \\
& =\frac{2}{\sigma T_{0}^{3}} e^{-\left|t-t^{\prime}\right| / \tau_{1}} \frac{\tau_{1}}{\tau}\left[1+\gamma_{N}\left(\frac{p}{p_{\mathrm{T}}}\right)^{4}\right] .
\end{aligned}
$$

Within the free-streaming approximation, we have two ranges of interest $p \ll p_{\mathrm{T}}$ and $p_{\mathrm{T}} \ll p<p_{\mathrm{L}}$. For the former we recover the spatially flat spectrum in (4.63) and for the latter we obtain

$$
\begin{aligned}
G_{1 Z_{\mathrm{TT}} Z_{\mathrm{TT}}} & =\frac{4 \tau}{\sigma T_{0}^{3}}\left(\frac{\tau_{1}}{\tau}\right)^{2}\left[1+\gamma_{N}\left(\frac{p}{p_{\mathrm{T}}}\right)^{4}\right] \delta\left(t-t^{\prime}\right) \\
& =\frac{4 \gamma_{N}}{\gamma_{\Sigma}^{2}} \frac{c_{V}}{p^{4}} \delta\left(t-t^{\prime}\right) .
\end{aligned}
$$

The previous analysis clearly shows a transition from a flat spectrum for $p \ll p_{\mathrm{T}}$ to a power law spectrum $p^{-4}$ for $p_{\mathrm{T}} \ll p<p_{\mathrm{L}}$.

\subsection{Entropy and EMT fluctuations}

Having found the tensor correlations in the free-streaming limit $(\tau \rightarrow \infty)$, we can easily derive the fluctuations in the viscous EMT, the energy density and the entropy density. Since for large wavelengths we recover the zeroth order propagators and therefore the Landau-Lifshitz expression for the fluctuations of $\Pi_{\mathrm{TT}}^{\mu \nu}$, cfr. eq. (4.65), we only quote the expression for the viscous EMT for $p_{\mathrm{T}} \ll p<p_{\mathrm{L}}$, which reads

$$
\left\langle\Pi_{\mathrm{TT}^{j k}}(p, t) \Pi_{\mathrm{TT}}^{l m}\left(q, t^{\prime}\right)\right\rangle=(2 \pi)^{3} \frac{16 \gamma_{N}}{3 \gamma_{\Sigma}^{2}} \frac{c_{V}^{3} \sigma^{2} T_{0}^{8}}{p^{4}} \delta(p+q) S_{\mathrm{TT}^{j k l m}}^{j p] \delta}\left(t-t^{\prime}\right)
$$


The contribution to the energy density in this model is

$$
\rho=\sigma T_{0}^{4}\left[1+\frac{7}{4} Z_{\mathrm{TT}}^{j k} Z_{\mathrm{TT} j k}\right] .
$$

To compute the expectation value of $Z_{\mathrm{TT}}{ }^{j k} Z_{\mathrm{TT} j k}$ in the equal time limit we use the full form eq. (5.47) to get

$$
\langle\rho\rangle=\sigma T_{0}^{4}\left[1+\frac{7}{\sigma T_{0}^{3}} \int \frac{d^{3} p}{(2 \pi)^{3}} \frac{1+\gamma_{N}\left(\frac{p}{p_{\mathrm{T}}}\right)^{4}}{1+\gamma_{\Sigma}\left(\frac{p}{p_{\mathrm{T}}}\right)^{4}}\right]
$$

The spectrum is flat for both regimes $p \ll p_{\mathrm{T}}$ and $p_{\mathrm{T}} \ll p<p_{\mathrm{L}}$, but in the latter the amplitude is diminished by a factor

$$
\frac{\gamma_{N}}{\gamma_{\Sigma}} \approx 0.47
$$

We may find the spectrum of entropy fluctuations in a similar way. The entropy density is

$$
s=\frac{4}{3} \sigma T_{0}^{3}\left[1+\frac{9}{8} Z_{\mathrm{TT}}{ }^{j k} Z_{\mathrm{TT} j k}\right] .
$$

Therefore

$$
\langle s\rangle=\frac{4}{3} \sigma T^{3}\left[1+\frac{9}{2 \sigma T_{0}^{3}} \int \frac{d^{3} p}{(2 \pi)^{3}} \frac{1+\gamma_{N}\left(\frac{p}{p_{\mathrm{T}}}\right)^{4}}{1+\gamma_{\Sigma}\left(\frac{p}{p_{\mathrm{T}}}\right)^{4}}\right]
$$

Of course, these are the spectra induced by irreducible tensor fluctuations only; the full energy and entropy spectra will also have contributions from vector modes.

These nontrivial spectra show that there is a definite redistribution of entropy among the short wavelength modes because of nonlinear effects. Although this equilibrium analysis does not allow us to determine the direction of the energy and entropy flows, the fact that when equilibrium is reached the short wavelength spectrum is depleted with respect to the linear case suggests the existence of an inverse cascade of entropy [102].

\section{Conclusions and discussion}

In this paper we began the study of the non-linear hydrodynamics of a real relativistic conformal fluid within the framework of Divergence Type Theories, which have the advantage that the Second Law of Thermodynamics is satisfied non-perturbatively.

In Second Order Theories such as DTTs, the fact that non-ideal effects are described by a new independent tensor variable permits to enlarge the set of hydrodynamic effects, as now quadrupolar oscillations represented by purely tensor modes are allowed in the flow, besides the scalar and vector ones already present in First Order theories.

This fact was previously exploited in [49] to investigate the induction of primordial gravitational waves by the presence of these modes in the Early Universe plasma, and also in [50] in the context of Early Universe magnetogenesis. 
In this manuscript we began to develop the nonlinear hydrodynamics of real relativistic fluids by studying in a self-consistent way thermally induced tensor fluctuations.

We consider a simple situation where tensor modes are excited by a Gaussian noise with a white spectrum. As was just said, this noise is due to the fluid own thermal fluctuations and the spectrum can be computed from the fluctuation-dissipation theorem.

From the analysis in subsection 3.1 this means that there appears an explicitly stochastic source in the conservation equation for the non-equilibrium current $A^{\mu \nu \rho}$, while the energy-momentum conservation equation is unmodified. This may be interpreted as if entropy is added to the system, while keeping constant its energy content.

Using techniques borrowed from Quantum Field Theory to study non-linear hydrodynamics, such as the Two-Particle-Irreducible Effective Action and the Martin-Siggia-Rose formalism, we wrote down the evolution equations for the retarded and Hadamard propagators for both the vector and tensor sectors. We first found the lowest linear order expression for the two-point functions and latter non-linear fluctuations around equilibrium were considered. It was found that the non-linearities renormalized the relaxation time of the theory in a way that induces a depletion of the tensor correlations in the range $p_{\mathrm{T}} \ll p \leq p_{\mathrm{L}}$ (eq. (5.45)), with $p_{\mathrm{L}}$ the largest value of $p$ for which the loop expansion is consistent. Stated otherwise, we found that tensor fluctuations have a flat spectrum for the largest scales, which turns to a power law $p^{-4}$ spectrum in the small length-scale sector.

In view of the exponentially decreasing time dependence of the two point functions, these corrections to the tensor fluctuation spectrum are significative for times shorter than the macroscopic relaxation time, or else on scales of the order $r<\tau$ which are the relevant ones at the initial stages of a heavy-ion collision.

Concerning the entropy to lowest order, the correction to the entropy density is quadratic in the fluctuations and consequently it is also diminished in the large $p$ range. This result suggests that tensor modes could sustain a turbulent inverse cascade of entropy [102], and we intend to study this issue in a forthcoming work.

Besides the studies mentioned just above, other systems where fluid tensor modes can play an important role are Neutron Stars [5-7] and Early Universe plasmas [8], to mention a few. In both systems, the fluids are non-ideal relativistic plasma. Therefore it is important to have a solid hydrodynamic theory in order to understand the features of those systems. This work is a small step toward that goal and sets the basis for more complete studies of tensor turbulence where energy injection can also be taken into account.

\section{A Scaling of the relevant diagrams}

In this appendix we discuss the scaling rules of the subset of diagrams that build the different propagators. We perform a general analysis in which we consider an arbitrary number of loops $L$ and find the condition over a particular combination of parameters that makes the loop expansion valid.

It is usual to draw the higher loop corrections in Quantum Field Theory as Feynman diagrams $[113,118]$. In any scheme the vertices correspond to the interaction action $S_{\text {int }}$ and the internal lines to the propagators. 
Let us take the noise kernel as an example. Diagrammatically the noise kernel has two external vertices of the same kind: $\left(Y_{\mathrm{TT}}-V-Z_{\mathrm{T}}\right)$ with $Y_{\mathrm{TT}}$ in the external lines. In order to simplify, we shall consider that in the internal structure of the noise kernel we just have the fields $Y, V$ and $Z_{\mathrm{T}}$ and vertices of the type $\left(Y-Z_{\mathrm{T}}-Z_{\mathrm{T}}\right)$ or $(Y-V-V)$. In this case the internal lines are $\langle V V\rangle,\langle V Y\rangle,\left\langle V Z_{\mathrm{T}}\right\rangle,\left\langle Z_{\mathrm{T}} Y\right\rangle$ and $\left\langle Z_{\mathrm{T}} Z_{\mathrm{T}}\right\rangle$.

Let us call the number of internal vertices $v$ and internal lines $j$ of the different types as $v(Y V V), v\left(Y Z_{\mathrm{T}} Z_{\mathrm{T}}\right), j(V V), j(V Y), j\left(V Z_{\mathrm{T}}\right), j\left(Z_{\mathrm{T}} Y\right)$ and $j\left(Z_{\mathrm{T}} Z_{\mathrm{T}}\right)$. If the number of loops is $L$ we must have the following constraints

$$
\begin{aligned}
2+2 v(Y V V) & =2 j(V V)+j(V Y)+j\left(V Z_{\mathrm{T}}\right) \\
2+2 v\left(Y Z_{\mathrm{T}} Z_{\mathrm{T}}\right) & =2 j\left(Z_{\mathrm{T}} Z_{\mathrm{T}}\right)+j\left(Z_{\mathrm{T}} Y\right)+j\left(V Z_{\mathrm{T}}\right) \\
v(Y V V)+v\left(Y Z_{\mathrm{T}} Z_{\mathrm{T}}\right) & =j(V Y)+j\left(Z_{\mathrm{T}} Y\right) \\
j(V V)+j\left(V Z_{\mathrm{T}}\right)+j\left(Z_{\mathrm{T}} Z_{\mathrm{T}}\right) & =L+1
\end{aligned}
$$

Every component of the structure (internal lines, internal and external vertices and loopintegrals) has a scaling factor. The complete diagram scales as the product of the scaling factors of each component. From tables 1 and 2 we extract the rules for the propagators, namely

$$
\begin{aligned}
\langle V V\rangle & \rightarrow C_{V V} \sim\left(\sigma T_{0}^{3}\right)^{-1} \\
\langle V Y\rangle & \rightarrow C_{V Y} \sim\left(\sigma T_{0}^{4}\right)^{-1} \\
\left\langle V Z_{\mathrm{T}}\right\rangle & \rightarrow C_{V Z_{\mathrm{T}}} \sim\left(\sigma T_{0}^{3} p\right)^{-1} \\
\left\langle Z_{\mathrm{T}} Y\right\rangle & \rightarrow C_{Z_{\mathrm{T}} Y} \sim\left(\sigma T_{0}^{4} p\right)^{-1} \\
\left\langle Z_{\mathrm{T}} Z_{\mathrm{T}}\right\rangle & \rightarrow C_{Z_{\mathrm{T}} Z_{\mathrm{T}}} \sim\left(\sigma T_{0}^{3} p^{2}\right)^{-1} .
\end{aligned}
$$

From eqs. (4.28) and (4.30) we get the vertex scaling

$$
\begin{aligned}
(Y V V) & \rightarrow V_{Y V V} \sim \sigma T_{0}^{4} p \\
\left(Y Z_{\mathrm{T}} Z_{\mathrm{T}}\right) & \rightarrow V_{Y Z_{\mathrm{T}} Z_{\mathrm{T}}} \sim \sigma T_{0}^{4} p^{3} .
\end{aligned}
$$

We must also add a factor of $p^{3}$ for each loop integral and a factor $1 / c_{V} p$ for each integral over the time labels attached to the internal vertices. We extract the singular part of the time-dependence, thereby introducing a new overall factor $\delta\left(t-t^{\prime}\right) /\left(c_{V} p\right)$. So finally the complete diagram scales as

$$
\delta\left(p-p^{\prime}\right) \delta\left(t-t^{\prime}\right) \frac{a^{2} T_{0}^{7} p}{c_{V} \sigma}\left(\frac{p^{3}}{c_{V}^{2} \sigma T_{0}^{3}}\right)^{L} .
$$

If we define $p_{\mathrm{L}}^{3}=c_{V}^{2} \sigma T_{0}^{3}$, the condition to make the loop expansion consistent reads $p<p_{\mathrm{L}}$.

\section{Acknowledgments}

N.M.G. thanks P. Minnini for fruitful discussions. The work of E.C. was supported in part by CONICET and Universidad de Buenos Aires. N.M.G. is supported by a fellowship of Universidad de Buenos Aires. A.K. acknowledges financial support from FAPESB grant FAPESB-PVE-015/2015/PET0013/2016, and support from Universidade Estadual de Santa Cruz. 
Open Access. This article is distributed under the terms of the Creative Commons Attribution License (CC-BY 4.0), which permits any use, distribution and reproduction in any medium, provided the original author(s) and source are credited.

\section{References}

[1] L.D. Landau, On multiple production of particles during collisions of fast particles, Izv. Akad. Nauk SSSR 17 (1953) 51.

[2] L.D. Landau and Z. Belenkij, A hydrodynamic theory of multiple formation of particles, Usp. Phys. Nauk SSSR 56 (1955) 309.

[3] P. Romatschke and U. Romatschke, Relativistic fluid dynamics in and out equilibrium Ten years of progress in theory and numerical simulations of nuclear collisions, Cambridge University Press (2019).

[4] M. Strickland, Anisotropic Hydrodynamics: Three lectures, Act. Phys. Pol. B 45 (2014) 2355.

[5] X.-G. Huang, M. Huang, D.H. Rischke and A. Sedrakian, Anisotropic Hydrodynamics, Bulk Viscosities and R-Modes of Strange Quark Stars with Strong Magnetic Fields, Phys. Rev. D 81 (2010) 045015 [arXiv:0910.3633] [INSPIRE].

[6] J.L. Friedman and N. Stergioulas, Rotating Relativistic Stars, Cambridge Monogr. Math. Phys., Cambridge University Press, New York, U.S.A. (2013).

[7] V. Paschalidis and N. Stergioulas, Rotating Stars in Relativity, Living Rev. Rel. 20 (2017) 7 [arXiv:1612.03050] [INSPIRE].

[8] P. Niksa, M. Schlederer and G. Sigl, Gravitational Waves produced by Compressible MHD Turbulence from Cosmological Phase Transitions, Class. Quant. Grav. 35 (2018) 144001 [arXiv: 1803.02271] [INSPIRE].

[9] W. Israel, Covariant fluid mechanics and thermodynamics: An introduction, in Relativistic Fluid Dynamics, A.M. Anile and Y. Choquet-Bruhat eds., Springer, New York, U.S.A. (1988).

[10] C. Eckart, The Thermodynamics of irreversible processes. III. Relativistic theory of the simple fluid, Phys. Rev. 58 (1940) 919 [INSPIRE].

[11] L.D. Landau and E.M. Lifshitz, Fluid Mechanics, Pergamon Press, Oxford, U.K. (1959).

[12] I. Müller, Zum Paradoxon der Wärmeleitungstheorie, Z. Phys. 198 (1967) 329 [InSPIRE].

[13] H. Grad, On the kinetic theory of rarefied gases, Commun. Pure Appl. Math. 2 (1949) 331.

[14] J.C. Maxwell, On the dynamical theory of gases, Phil. Trans. Roy. Soc. Lond. 157 (1867) 49.

[15] C. Cattaneo, Sulla conduzione del calore, Atti Sem. Mat. Fis. Univ. Modena 3 (1948) 3.

[16] C. Cattaneo, Sur la propagation de la chaleur en relativité, C.R. Acad. Sci. Paris $\mathbf{2 4 7}$ (1958) 431.

[17] D.D. Joseph and L. Preziosi, Heat waves, Rev. Mod. Phys. 61 (1989) 41 [inSPIRE].

[18] W. Israel, Nonstationary irreversible thermodynamics: A Causal relativistic theory, Annals Phys. 100 (1976) 310 [inSPIRE]. 
[19] W. Israel and J.M. Stewart, Thermodynamics of nonstationary and transient effects in a relativistic gas, Phys. Lett. A 58 (1976) 213.

[20] W. Israel and J.M. Stewart, Transient relativistic thermodynamics and kinetic theory, Annals Phys. 118 (1979) 341 [INSPIRE].

[21] W. Israel and M. Stewart, On transient relativistic thermodynamics and kinetic theory. II, Proc. Roy. Soc. Lond. A 365 (1979) 43.

[22] W. Israel and J.M. Stewart, Progress in relativistic thermodynamics and electrodynamics of continuous media, in General Relativity and Gravitation, vol. 2, A. Held ed., Plenum, New York, p. 491 (1980).

[23] W.A. Hiscock and L. Lindblom, Generic instabilities in first-order dissipative relativistic fluid theories, Phys. Rev. D 31 (1985) 725 [InSPIRE].

[24] W.A. Hiscock and L. Lindblom, Stability in dissipative relativistic fluid theories, Contemp. Math. 71 (1988) 181.

[25] W.A. Hiscock and L. Lindblom, Nonlinear pathologies in relativistic heat-conducting fluid theories, Phys. Lett. A 131 (1988) 509.

[26] T.S. Olson, Stability and causality in the Israel-Stewart energy frame theory, Annals Phys. 199 (1990) 18 [INSPIRE].

[27] T.S. Olson and W.A. Hiscock, Plane steady shock waves in Israel-Stewart fluids, Annals Phys. 204 (1990) 331 [INSPIRE].

[28] D. Jou, J.M. Rubi and J. Casas-Vázquez, Hydrodynamical fluctuations in extended irreversible thermodynamics, Physica A 101 (1980) 588.

[29] D. Pavón, D. Jou and J. Casas-Vázquez, Heat conduction in relativistic thermodynamics, J. Phys. A 13 (1980) L77.

[30] D. Jou, J. Casas-Vázquez and G. Lebon, Extended irreversible thermodynamics, Springer, Berlin, Germany (2010).

[31] F.S. Bemfica, M.M. Disconzi and J. Noronha, Causality of the Einstein-Israel-Stewart Theory with Bulk Viscosity, Phys. Rev. Lett. 122 (2019) 221602 [arXiv:1901.06701] [INSPIRE].

[32] A.M. Anile, D. Pavón and V. Romano, The Case for hyperbolic theories of dissipation in relativistic fluids, gr-qc/9810014 [INSPIRE].

[33] L. Herrera and D. Pavón, Why hyperbolic theories of dissipation cannot be ignored: Comments on a paper by Kostädt and Liu, Phys. Rev. D 64 (2001) 088503 [gr-qc/0102026] [INSPIRE].

[34] A. Muronga, Causal theories of dissipative relativistic fluid dynamics for nuclear collisions, Phys. Rev. C 69 (2004) 034903 [nucl-th/0309055] [INSPIRE].

[35] M. Aguilar and E. Calzetta, Causal Relativistic Hydrodynamics of Conformal Fermi-Dirac Gases, Phys. Rev. D 95 (2017) 076022 [arXiv: 1701.01916] [InSPIRE].

[36] C. Gale, S. Jeon and B. Schenke, Hydrodynamic Modeling of Heavy-Ion Collisions, Int. J. Mod. Phys. A 28 (2013) 1340011 [arXiv:1301.5893] [INSPIRE].

[37] S. Jeon and U. Heinz, Introduction to Hydrodynamics, Int. J. Mod. Phys. E 24 (2015) 1530010 [arXiv: 1503.03931] [INSPIRE]. 
[38] I.S. Liu, I. Müller and T. Ruggeri, Relativistic thermodynamics of gases, Annals Phys. 169 (1986) 191.

[39] R.P. Geroch and L. Lindblom, Dissipative relativistic fluid theories of divergence type, Phys. Rev. D 41 (1990) 1855 [INSPIRE].

[40] R. Geroch and L. Lindblom, Causal theories of dissipative relativistic fluids Annals Phys. 207 (1991) 394.

[41] O.A. Reula and G.B. Nagy, On the causality of a dilute gas as a dissipative relativistic fluid theory of divergence type, J. Phys. A 28 (1995) 6943.

[42] J. Peralta-Ramos and E. Calzetta, Divergence-type nonlinear conformal hydrodynamics, Phys. Rev. D 80 (2009) 126002 [arXiv:0908.2646] [InSPIRE].

[43] J. Peralta-Ramos and E. Calzetta, Divergence-type $2+1$ dissipative hydrodynamics applied to heavy-ion collisions, Phys. Rev. C 82 (2010) 054905 [arXiv: 1003.1091] [InSPIRE].

[44] E. Calzetta, Hydrodynamic approach to boost invariant free streaming, Phys. Rev. D 92 (2015) 045035 [arXiv:1402.5278] [INSPIRE].

[45] E. Calzetta, Relativistic fluctuating hydrodynamics, Class. Quant. Grav. 15 (1998) 653 [gr-qc/9708048] [INSPIRE].

[46] L. Lehner, O.A. Reula and M.E. Rubio, Hyperbolic theory of relativistic conformal dissipative fluids, Phys. Rev. D 97 (2018) 024013 [arXiv: 1710.08033] [InSPIRE].

[47] E. Calzetta and L. Cantarutti, Dissipative type theories for Bjorken and Gubser flows, Int. J. Mod. Phys. A 35 (2020) 2050074 [arXiv:1912.10562] [InSPIRE].

[48] R. Loganayagam, Entropy Current in Conformal Hydrodynamics, JHEP 05 (2008) 087 [arXiv:0801.3701] [INSPIRE].

[49] N. Mirón-Granese and E. Calzetta, Primordial gravitational waves amplification from causal fluids, Phys. Rev. D 97 (2018) 023517 [arXiv:1709.01661] [INSPIRE].

[50] E. Calzetta and A. Kandus, A Hydrodynamic Approach to the Study of Anisotropic Instabilities in Dissipative Relativistic Plasmas, Int. J. Mod. Phys. A 31 (2016) 1650194 [arXiv: 1602.01622] [INSPIRE].

[51] L.D. Landau and E.M. Lifshitz, Hydrodynamic Fluctuations, Zh. Eksp. Teor. Fiz 32 (1957) 618 [Sov. Phys. JETP 5 (1957) 512)].

[52] L.D. Landau and E.M. Lifshitz, Statistical Mechanics, part II, Pergamon Press, Oxford, U.K. (1959).

[53] R. Fox and G. Uhlembeck, Contributions to Non-Equilibrium Thermodynamics. I. Theory of Hydrodynamical Fluctuations, Phys. Fluids 13 (1970) 1893.

[54] R. Fox and G. Uhlembeck, Contributions to Nonequilibrium Thermodynamics. II. Fluctuation Theory for the Boltzmann Equation, Phys. Fluids 13 (1970) 2881.

[55] H.B. Callen and T.A. Welton, Irreversibility and generalized noise, Phys. Rev. 83 (1951) 34 [INSPIRE].

[56] G.D. Moore and K.A. Sohrabi, Kubo Formulae for Second-Order Hydrodynamic Coefficients, Phys. Rev. Lett. 106 (2011) 122302 [arXiv:1007.5333] [InSPIRE].

[57] P. Kovtun, G.D. Moore and P. Romatschke, The stickiness of sound: An absolute lower limit on viscosity and the breakdown of second order relativistic hydrodynamics, Phys. Rev. D 84 (2011) 025006 [arXiv:1104.1586] [INSPIRE]. 
[58] J. Peralta-Ramos and E. Calzetta, Shear viscosity from thermal fluctuations in relativistic conformal fluid dynamics, JHEP 02 (2012) 085 [arXiv: 1109.3833] [INSPIRE].

[59] B.J. Alder and T.E. Wainwright, Decay of the Velocity Autocorrelation Function, Phys. Rev. A 1 (1970) 18 [INSPIRE].

[60] E.M.H. Hauge and E.H. van Leeuwen, Asymptotic time behavior of correlation functions, Phys. Rev. Lett. 25 (1970) 1254.

[61] P. Kovtun and L.G. Yaffe, Hydrodynamic fluctuations, long time tails and supersymmetry, Phys. Rev. D 68 (2003) 025007 [hep-th/0303010] [INSPIRE].

[62] J.I. Kapusta, B. Müller and M. Stephanov, Relativistic Theory of Hydrodynamic Fluctuations with Applications to Heavy Ion Collisions, Phys. Rev. C 85 (2012) 054906 [arXiv:1112.6405] [INSPIRE].

[63] Y. Akamatsu, A. Mazeliauskas and D. Teaney, A kinetic regime of hydrodynamic fluctuations and long time tails for a Bjorken expansion, Phys. Rev. C 95 (2017) 014909 [arXiv: 1606.07742] [INSPIRE].

[64] M. Martinez and T. Schäfer, Stochastic hydrodynamics and long time tails of an expanding conformal charged fluid, Phys. Rev. C 99 (2019) 054902 [arXiv: 1812.05279] [InSPIRE].

[65] X. An, G. Basar, M. Stephanov and H.-U. Yee, Relativistic Hydrodynamic Fluctuations, Phys. Rev. C 100 (2019) 024910 [arXiv: 1902.09517] [INSPIRE].

[66] A. Kandus, E.A. Calzetta, F.D. Mazzitelli and C.E.M. Wagner, Cosmological magnetic fields from gauge mediated supersymmetry breaking models, Phys. Lett. B 472 (2000) 287 [hep-ph/9908524] [INSPIRE].

[67] E.A. Calzetta and A. Kandus, Selfconsistent estimates of magnetic fields from reheating, Phys. Rev. D 65 (2002) 063004 [astro-ph/0110341] [INSPIRE].

[68] E. Calzetta and E. Verdaguer, Noise induced transitions in semiclassical cosmology, Phys. Rev. D 59 (1999) 083513 [gr-qc/9807024] [INSPIRE].

[69] E. Calzetta, A. Roura and E. Verdaguer, Dissipation, noise and vacuum decay in quantum field theory, Phys. Rev. Lett. 88 (2002) 010403 [hep-ph/0101052] [INSPIRE].

[70] E. Hopf, Statistical hydromechanics and functional analysis, J. Rat. Mech. Anal. 1 (1952) 87.

[71] R.M. Lewis and R.H. Kraichnan, A space-time functional formalism for turbulence, Commun. Pure Appl. Math. 15 (1962) 397.

[72] C. De Dominicis and P.C. Martin, Energy spectra of certain randomly-stirred fluids, Phys. Rev. A 19 (1979) 419.

[73] S.s. Grozdanov and J. Polonyi, Viscosity and dissipative hydrodynamics from effective field theory, Phys. Rev. D 91 (2015) 105031 [arXiv:1305.3670] [INSPIRE].

[74] M. Crossley, P. Glorioso and H. Liu, Effective field theory of dissipative fluids, JHEP 09 (2017) 095 [arXiv: 1511.03646] [INSPIRE].

[75] K. Jensen, N. Pinzani-Fokeeva and A. Yarom, Dissipative hydrodynamics in superspace, JHEP 09 (2018) 127 [arXiv: 1701.07436] [INSPIRE].

[76] F.M. Haehl, R. Loganayagam and M. Rangamani, Effective Action for Relativistic Hydrodynamics: Fluctuations, Dissipation and Entropy Inflow, JHEP 10 (2018) 194 [arXiv: 1803.11155] [INSPIRE]. 
[77] P.C. Martin, E.D. Siggia and H.A. Rose, Statistical Dynamics of Classical Systems, Phys. Rev. A 8 (1973) 423 [inSPIRE].

[78] J. De Dominicis, Techniques de renormalisation de la théorie des champs et dynamique des phénomènes critiques, J. Physique Coll. 37 (1976) C1.

[79] A. Kamenev, Field Theory of Non-Equilibrium Systems, Cambridge University Press, Cambridge, U.K. (2011).

[80] G.L. Eyink, Turbulence Noise, J. Stat. Phys. 83 (1996) 955.

[81] J. Zanella and E. Calzetta, Renormalization group and nonequilibrium action in stochastic field theory, Phys. Rev. E 66 (2002) 036134 [cond-mat/0203566] [InSPIRE].

[82] W.D. McComb, Homogeneous, Isotropic Turbulence, Oxford University Press, Oxford, U.K. (2014).

[83] H.B.F. Nielsen, Small Representation Principle, Bled Workshops Phys. 14 (2013) 143 [arXiv: 1403.1407] [INSPIRE].

[84] L.L. Lee, A formulation of the theory of isotropic hydromagnetic turbulence in an incompressible fluid, Annals Phys. 32 (1965) 292.

[85] W.D. McComb, The Physics of Fluid Turbulence, Oxford University Press, Oxford, U.K. (1990).

[86] E. Calzetta, Kadanoff-Baym Equations for Near-Kolmogorov Turbulence, arXiv:0908.4068.

[87] P. Kovtun, Lectures on hydrodynamic fluctuations in relativistic theories, J. Phys. A 45 (2012) 473001 [arXiv:1205.5040] [INSPIRE].

[88] P. Kovtun, G.D. Moore and P. Romatschke, Towards an effective action for relativistic dissipative hydrodynamics, JHEP 07 (2014) 123 [arXiv:1405.3967] [INSPIRE].

[89] M. Harder, P. Kovtun and A. Ritz, On thermal fluctuations and the generating functional in relativistic hydrodynamics, JHEP 07 (2015) 025 [arXiv: 1502.03076] [INSPIRE].

[90] N. Andersson and G.L. Comer, Relativistic fluid dynamics: Physics for many different scales, Living Rev. Rel. 10 (2007) 1 [gr-qc/0605010] [InSPIRE].

[91] M.P. Heller and M. Spaliński, Hydrodynamics Beyond the Gradient Expansion: Resurgence and Resummation, Phys. Rev. Lett. 115 (2015) 072501 [arXiv: 1503.07514] [INSPIRE].

[92] P. Romatschke, Relativistic Hydrodynamic Attractors with Broken Symmetries: Non-Conformal and Non-Homogeneous, JHEP 12 (2017) 079 [arXiv:1710.03234] [INSPIRE].

[93] M. Strickland, J. Noronha and G. Denicol, Anisotropic nonequilibrium hydrodynamic attractor, Phys. Rev. D 97 (2018) 036020 [arXiv:1709.06644] [INSPIRE].

[94] G.S. Denicol and J. Noronha, Exact hydrodynamic attractor of an ultrarelativistic gas of hard spheres, Phys. Rev. Lett. 124 (2020) 152301 [arXiv:1908.09957] [INSPIRE].

[95] P. Ván and T.S. Biró, First order and stable relativistic dissipative hydrodynamics, Phys. Lett. B 709 (2012) 106 [arXiv:1109.0985] [INSPIRE].

[96] P. Kovtun, First-order relativistic hydrodynamics is stable, JHEP 10 (2019) 034 [arXiv: 1907.08191] [INSPIRE]. 
[97] A. Das, W. Florkowski, J. Noronha and R. Ryblewski, Equivalence between first-order causal and stable hydrodynamics and Israel-Stewart theory for boost-invariant systems with a constant relaxation time, Phys. Lett. B 806 (2020) 135525 [arXiv: 2001. 07983] [InSPIRE].

[98] A.L. García-Perciante, M.E. Rubio and O.A. Reula, Generic instabilities in the relativistic Chapman-Enskog heat conduction law, J. Stat. Phys. (2020) [arXiv:1908.04445] [inSPIRE].

[99] S. Weinberg, Cosmology, Oxford University Press, New York (2008).

[100] C. Caprini and D.G. Figueroa, Cosmological Backgrounds of Gravitational Waves, Class. Quant. Grav. 35 (2018) 163001 [arXiv:1801.04268] [INSPIRE].

[101] S. Kamata, M. Martinez, P. Plaschke, S. Ochsenfeld and S. Schlichting, Hydrodynamization and non-equilibrium Green's functions in kinetic theory, arXiv:2004.06751 [INSPIRE].

[102] G.L. Eyink and T.D. Drivas, Cascades and Dissipative Anomalies in Relativistic Fluid Turbulence, Phys. Rev. X 8 (2018) 011023 [arXiv: 1704.03541] [InSPIRE].

[103] E. Calzetta, Real relativistic fluids in heavy ion collisions, in 8th Summer School on Geometric, Algebraic and Topological Methods for Quantum Field Theory, pp. 155-191 (2013) [DOI] [arXiv:1310.0841] [INSPIRE].

[104] J.L. Anderson and H.R. Witting, A relativistic relaxation-time model for the Boltzmann equation, Physica 74 (1974) 466.

[105] J.L. Anderson and H.R. Witting, Relativistic quantum transport coefficients, Physica 74 (1974) 489.

[106] G. Policastro, D.T. Son and A.O. Starinets, The Shear viscosity of strongly coupled $N=4$ supersymmetric Yang-Mills plasma, Phys. Rev. Lett. 87 (2001) 081601 [hep-th/0104066] [INSPIRE].

[107] I. Müller, Speeds of propagation in classical and relativistic extended thermodynamics, Living Rev. Rel. 2 (1999) 1 [INSPIRE].

[108] K. Murase, Causal hydrodynamic fluctuations in non-static and inhomogeneous backgrounds, Annals Phys. 411 (2019) 167969.

[109] A. Kurkela, A. Mazeliauskas, J.-F. Paquet, S. Schlichting and D. Teaney, Effective kinetic description of event-by-event pre-equilibrium dynamics in high-energy heavy-ion collisions, Phys. Rev. C 99 (2019) 034910 [arXiv:1805.00961] [inSPIRE].

[110] A. Kurkela, A. Mazeliauskas, J.-F. Paquet, S. Schlichting and D. Teaney, Matching the Nonequilibrium Initial Stage of Heavy Ion Collisions to Hydrodynamics with QCD Kinetic Theory, Phys. Rev. Lett. 122 (2019) 122302 [arXiv:1805.01604] [INSPIRE].

[111] D. Förster, D.R. Nelson and M.J. Stephen, Large-distance and long-time properties of a randomly stirred fluid, Phys. Rev. A 16 (1977) 732 [inSPIRE].

[112] J. Zinn-Justin, Quantum field theory and critical phenomena, 3rd ed., Clarendon Press, Oxford, U.K. (1996).

[113] E.A. Calzetta and B.L. Hu, Nonequilibrium Quantum Field Theory, Cambridge University Press, Cambridge, U.K. (2008).

[114] R. Baier, P. Romatschke, D.T. Son, A.O. Starinets and M.A. Stephanov, Relativistic viscous hydrodynamics, conformal invariance and holography, JHEP 04 (2008) 100 [arXiv:0712.2451] [INSPIRE]. 
[115] G.S. Denicol, H. Niemi, E. Molnar and D.H. Rischke, Derivation of transient relativistic fluid dynamics from the Boltzmann equation, Phys. Rev. D 85 (2012) 114047 [Erratum ibid. 91 (2015) 039902] [arXiv: 1202.4551] [INSPIRE].

[116] P. Ramond, Field Theory: A Modern Primer, Frontiers in Physics, Avalon Publishing, New York, U.S.A. (1997).

[117] H. Kleinert and V. Schulte-Frohlinde, Critical Properties of $\phi^{4}$-theories, World Scientific, Singapore (2001).

[118] M.E. Peskin and D.V. Schroeder, An Introduction to Quantum Field Theory, Addison-Wesley Pub. Co., Massachusetts, U.S.A. (1995). 